\title{
Mahler measure and entropy for commuting automorphisms of compact groups
}

\author{
Douglas Lind $^{1, \star}$, Klaus Schmidt ${ }^{2, \star}$, and Tom Ward ${ }^{2, \star}$ \\ 1 Department of Mathematics, University of Washington, Seattle, WA 98195, USA \\ 2 Mathematics Institute, University of Warwick, Coventry CV4 7AL, UK
}

\section{Summary}

We compute the joint entropy of $d$ commuting automorphisms of a compact metrizable group. Let $R_{d}=\mathbb{Z}\left[u_{1}^{ \pm 1}, \ldots, u_{d}^{ \pm 1}\right]$ be the ring of Laurent polynomials in $d$ commuting variables, and $M$ be an $R_{d}$-module. Then the dual group $X_{M}$ of $M$ is compact, and multiplication on $M$ by each of the $d$ variables corresponds to an action $\alpha_{M}$ of $\mathbb{Z}^{d}$ by automorphisms of $X_{M}$. Every action of $\mathbb{Z}^{d}$ by automorphisms of a compact abelian group arises this way. If $f \in R_{d}$, our main formula shows that the topological entropy of $\alpha_{R_{d} /\langle f\rangle}$ is given by

$$
h\left(\alpha_{R_{d}\langle f f}\right)=\log \mathrm{M}(f)=\int_{0}^{1} \ldots \int_{0}^{1} \log \left|f\left(e^{2 \pi i t_{1}}, \ldots, e^{2 \pi i t_{d}}\right)\right| d t_{1} \ldots d t_{d},
$$

where $M(f)$ is the Mahler measure of $f$. This reduces to the classical result for toral automorphisms via Jensen's formula. While the entropy of a single automorphism of a compact group is always the logarithm of an algebraic integer, this no longer seems to hold for joint entropy of commuting automorphisms since values such as $7 \zeta(3) / 4 \pi^{2}$ occur. If $\mathfrak{p}$ is a non-principal prime ideal, we show $h\left(\alpha_{R_{d} / p}\right)=0$. Using an analogue of the Yuzvinskii-Thomas addition formula, we compute $h\left(\alpha_{M}\right)$ for arbitrary $R_{d}$-modules $M$, and then the joint entropy for an action of $\mathbb{Z}^{d}$ on a (not necessarily abelian) compact group.

Using a result of Boyd, we characterize those $\alpha_{M}$ which have completely positive entropy in terms of the prime ideals associated to $M$, and show this condition implies that $\alpha_{M}$ is mixing of all orders. We also establish an analogue of Berg's theorem, proving that if $\alpha_{M}$ has finite entropy then Haar measure is the unique measure of maximal entropy if and only if $\alpha_{M}$ has completely positive entropy. Finally, we show that for expansive actions the growth rate of the number of periodic points equals the topological entropy.

* The authors gratefully acknowledge support from NSF Grant DMS-8706284, the IBM Thomas J. Watson Research Center, the Milliman Endowment, and SERC Award B85318868 


\section{§1. Introduction}

The main purpose of this paper is to compute the joint topological entropy of $d$ commuting automorphisms of a compact metrizable group. For a single automorphism this computation was carried out in a series of papers in the 1960's (cf. [LW, §1]), culminating in Yuzvinskii's general result [Yz2].

Recently, Kitchens and Schmidt [KS1] have established a general framework for the study of commuting automorphisms of compact groups. To describe this, suppose $\alpha$ is an action of $\mathbb{Z}^{d}$ by automorphisms of a compact abelian group $X$. Let $R_{\mathrm{d}}=\mathbb{Z}\left[u_{d}^{ \pm 1}, \ldots, u_{\mathrm{d}}^{ \pm 1}\right]$ be the ring of Laurent polynomials in $d$ commuting variables. In $\S 2$ we show how $\alpha$ induces an $R_{d}$-module structure on the dual group $M$ of $X$. Conversely, if $M$ is an $R_{d}$-module, then there is a natural induced $\mathbb{Z}^{d}$-action $\alpha_{M}$ on its compact dual $X_{M}$. The interplay of the dynamical properties of $\alpha_{M}$ on the one hand, and the algebraic properties of $M$ on the other, gives this point of view its particular interest.

Suppose $f=f\left(u_{1}, \ldots, u_{d}\right) \in R_{d}$, and let $\mathfrak{f}=\langle f\rangle$ be the principal ideal generated by $f$. Our main formula computes the topological entropy of the $\mathbb{Z}^{d}$-action corresponding to the $R_{d}$-module $R_{d} / \mathfrak{f}$. We show in Theorem 3.1 that

$$
h\left(\alpha_{R_{d} / f}\right)=\log M(f)=\int_{0}^{1} \ldots \int_{0}^{1} \log \left|f\left(e^{2 \pi i t_{1}}, \ldots, e^{2 \pi i t_{d}}\right)\right| d t_{1} \ldots d t_{d} .
$$

Here $\mathrm{M}(f)$ is the Mahler measure of $f$, introduced by Mahler [Mh1], [Mh2] in connection with inequalities for coefficients of polynomials in several variables. Smyth [Sm2] has computed the integral in $(1-1)$ for some polynomials. For example,

$$
h\left(\alpha_{R_{2} /\langle 1+u+v\rangle}\right)=\frac{3 \sqrt{3}}{4 \pi} \sum_{n=1}^{\infty}\left(\frac{n}{3}\right) \frac{1}{n^{2}} \cong 0.3230659472,
$$

where $\left(\frac{n}{3}\right)$ is the Legendre symbol, and

$$
h\left(\alpha_{R_{3} /\langle 1+u+v+w\rangle}\right)=\frac{7 \zeta(3)}{4 \pi^{2}} \cong 0.2131391994,
$$

where $\zeta$ is the Riemann zeta function.

The formula (1-1) reduces via Jensen's formula to Yuzvinskii's result when $d=1$.

We devote $\S 3$ to showing (1-1). The proof that $h\left(\alpha_{R_{d} / f}\right) \geqq \log M(f)$ uses a lower estimate for entropy involving 1-dimensional integrals that Lawton [Lw] has shown converge to $\log M(f)$. For the opposite inequality another kind of estimate is used, yielding an upper bound for $h\left(\alpha_{R_{d} / f}\right)$ that is a Riemann sum approximation to the integral in (1-1). Since the integrand may not be continuous, a proof that these Riemann sums converge to the integral is required. This is supplied in Lemma 3.5 , where we show using perturbation arguments that the limit defining the Bowen entropy for a linear map is uniform on compact sets of linear maps.

For a non-principal prime ideal $p$ in $R_{d}$, we prove in Theorem 4.2 that $h\left(\alpha_{R_{d} / p}\right)=0$. In Appendix B we supply a proof of the "addition formula," which shows that if $N$ is a submodule of $M$, then $h\left(\alpha_{M}\right)=h\left(\alpha_{N}\right)+h\left(\alpha_{M / N}\right)$. If $M$ is a 
noetherian $R_{d}$-module, then by Lemma 4.3 there are submodules

$$
0=M_{0} \subset M_{1} \subset \ldots \subset M_{r-1} \subset M_{r}=M
$$

with $M_{j} / M_{j-1} \cong R_{d} / \mathfrak{p}_{j}$, where $\mathfrak{p}_{j}$ is a prime ideal. By the addition formula we then have

$$
h\left(\alpha_{M}\right)=\sum_{j=1}^{r} h\left(\alpha_{\left.R_{d} / p\right)}\right),
$$

where each summand has been computed. We continue $\S 4$ with the calculation in Theorem 4.5 of $h(\alpha)$ for a $\mathbb{Z}^{d}$-action $\alpha$ on a (not necessarily abelian) compact group. Whether the set of values $h(\alpha)$ for such actions is countable, or all of $[0, \infty]$, is shown in Theorem 4.6 to be equivalent to a problem of Lehmer about algebraic integers that has been open for fifty years.

A polynomial in $R_{d}$ is called generalized cyclotomic if it can be written in the form $\Phi\left(u_{1}^{n_{1}} u_{2}^{n_{2}} \ldots u_{d}^{n_{d}}\right)$, where the $n_{j} \in \mathbb{Z}$ and $\Phi(u)$ is a cyclotomic polynomial in one variable. A theorem of Boyd [By1] implies that $h\left(\alpha_{R_{d} / f}\right)=0$ if and only if $f$ is a monomial times a product of generalized cyclotomic polynomials. This and other examples and remarks are collected in $\$ 5$.

Our characterization of zero entropy is used in Theorem 6.5 to obtain a complete description of the Pinsker $\sigma$-subalgebra for $\alpha_{M}$. In particular, $\alpha_{M}$ has completely positive entropy if and only if every prime ideal associated to $M$ is principal but not generated by a generalized cyclotomic polynomial. In this case, a result of Kaminski $[\mathrm{Km}]$ shows that $\alpha_{M}$ is mixing of all orders, providing a partial answer to a question raised by one of us [ $\mathrm{Sc} 1$, Problem 3.9]. We next give a more careful analysis of the properties of $\alpha_{M}$ that can be deduced from the prime ideals $\mathfrak{p}_{j}$ occurring in a given filtration (1-2) of $M$. In particular, we show in Propostion 6.10 that the minimal prime ideals associated to $M$ must occur, and with the same multiplicity, in every filtration. Consequently, we obtain in Theorem 6.13 a computable sufficient condition for $\alpha_{M}$ to have completely positive entropy. Our analysis also yields an analogue of Berg's theorem on the unique ergodicity of group automorphisms $[\mathrm{Bg}]$. We prove in Theorem 6.14 that if $h\left(\alpha_{M}\right)<\infty$, then Haar measure on $X_{M}$ is the unique measure of maximal entropy if and only if $\alpha_{M}$ has completely positive entropy.

In certain situations the growth rate of the number of periodic points gives the topological entropy. Although examples show that this fails to hold in general, we show in Theorem 7.1 that it holds for all expansive actions. The proof uses the characterization of expansiveness of $\alpha_{M}$ in terms of the varieties of the prime ideals associated to $M$ given in [Sc2, Thm. 3.9].

We are grateful to David Boyd and Robert Warfield for many useful conversations.

\section{§2. Notations and conventions}

We describe here some of the notations and machinery used throughout this paper.

The symbols $\mathbb{Z}, \mathbb{R}$, and $\mathbb{C}$ denote the integers, reals, and complex numbers, respectively. Let $\mathbb{T}$ denote the additive circle group $\mathbb{R} / \mathbb{Z}$, and $\mathbb{S}=\{z \in \mathbb{C}:|z|=1\}$ be 
its multiplicative counterpart. For clarity, $d$-tuples will be denoted by boldface letters. If $\mathbf{u}=\left(u_{1}, \ldots, u_{d}\right)$ and $\mathbf{r}=\left(r_{1}, \ldots, r_{d}\right)$, we put $\mathbf{u}^{r}=u_{1}^{r_{1}} u_{2}^{r_{2}} \ldots u_{d}^{r_{d}}$. For a scalar $u$, let $u^{\mathrm{r}}$ denote $\left(u^{r_{1}}, \ldots, u^{r_{d}}\right)$.

A rectangle in $\mathbb{Z}^{d}$ is a set of the form $Q=\prod_{j=1}^{d}\left\{b_{j}, \ldots, b_{j}+l_{j}-1\right\}$. Its girth is defined to be $g(Q)=\min _{1 \leqq j \leqq d} l_{j}$.

All groups that we consider will be metrizable. $\mathrm{A} \mathbb{Z}^{\mathrm{d}}$-action $\alpha$ on a group $X$ is a homomorphism $\alpha$ of $\mathbb{Z}^{d}$ to be the group of continuous (algebraic) automorphisms of $X$. The image of $\mathbf{n} \in \mathbb{Z}^{d}$ under $\alpha$ will be denoted by $\alpha^{\mathbf{n}}$. If $\alpha$ is a $\mathbb{Z}^{d}$-action and $A$ : $\mathbb{Z}^{d} \rightarrow \mathbb{Z}^{d}$ is a homomorphism, define the action $\alpha^{A}$ by $\left(\alpha^{A}\right)^{\mathrm{n}}=\alpha^{A \mathrm{n}}$.

Let $\alpha$ be a $\mathbb{Z}^{d}$-action on a compact abelian group $X$, and let $M$ be the discrete dual group of $X$. Since $X$ is metrizable and compact, it follows that $M$ is countable and discrete. If $\mathbf{e}_{j}$ denotes the $j$ th standard basis vector of $\mathbb{Z}^{d}$, then the automorphisms $\alpha^{\mathbf{e}}$, correspond under duality to commuting automorphisms $\beta_{j}$ of $M$. If $R_{d}$ denotes the ring $\mathbb{Z}\left[u_{1}^{ \pm 1}, \ldots, u_{d}^{ \pm 1}\right]$, then $M$ becomes an $R_{d}$-module under the action $u_{j} \cdot m=\beta_{j}(m)$ for $m \in M$. Conversely, if $M$ is a countable $R_{d}$-module, then multiplication by each of the $u_{j}$ gives $d$ commuting automorphisms of $M$. These transform to a $\mathbb{Z}^{d}$-action $\alpha_{M}$ on the compact metrizable dual group $X_{M}$ of $M$.

For a $\mathbb{Z}^{d}$-action $\alpha$ on a compact group $X$, let $h(\alpha)$ denote its topological entropy. Since we have not found a satisfactory treatment in the literature, we outline in Appendix A the steps needed to extend the theory of entropy to $d>1$. This includes showing that five ways to obtain $h(\alpha)$ agree. In particular, $h(\alpha)$ is the entropy of $\alpha$ as a measure-preserving action of $\mathbb{Z}^{d}$ on the Lebesgue space $\left(X, \mu_{X}\right)$, where $\mu_{X}$ is normalized Haar measure. The general theory of measure-preserving $\mathbb{Z}^{d}$-actions has been developed by Conze [C], to which we refer the reader for details and notation. We shall use Rohlin's theory of measurable partitions of a Lebesgue space. An excellent account of this theory is contained in Parry's book [P].

If $f \in R_{d}$, then the Mahler measure of $f$ is defined as

$$
M(f)=\exp \left[\int_{\mathbb{S}^{d}} \log |f(\mathbf{s})| d \mathbf{s}\right],
$$

where $d \mathrm{~s}$ is Haar measure on $\mathbb{S}^{d}$. This quantity can be regarded as the geometric mean of $f$ over the torus $\mathbb{S}^{d}$. If $f \neq 0$, then $1 \leqq M(f)<\infty$. It will be convenient here to make the convention that $M(0)=\infty$.

\section{§3. Entropy and Mahler measure}

Let $f \in R_{d}$, and $\mathfrak{f}=\langle f\rangle$ denote the principal ideal generated by $f$. Then $R_{d} / \mathfrak{f}$ is an $R_{d}$-module. In this section we compute the entropy of the induced $\mathbb{Z}^{d}$-action $\alpha_{R_{d} / f}$. Recall our convention that $M(0)=\infty$.

Theorem 3.1. If $f \in R_{d}$ and $\mathfrak{f}=\langle f\rangle$, then

$$
h\left(\alpha_{R_{d} / f}\right)=\log \mathrm{M}(f) \text {, }
$$

where $M(f)$ is the Mahler measure of $f$. 
Proof. First assume that $d=1$. We will show that (3-1) transforms via Jensen's formula to the classical entropy formula for toral and solenoidal automorphisms. We may assume without loss that $f(u)=c_{m} u^{m}+\ldots+c_{1} u+c_{0}$, where $c_{j} \in \mathbb{Z}$ and $c_{m} c_{0} \neq 0$. Kitchens and Schmidt [KS1] showed that $\alpha_{R_{1} / /}$ is isomorphic to the automorphism of an $m$-dimensional solenoid determined by the companion matrix of $f$. The entropy of such automorphisms has been computed by Yuzvinskii [Yz2] (see also [LW] for a short proof from a different viewpoint). Let $f(u)$ factor over $\mathbb{C}$ as $c_{m} \prod_{j=1}^{m}\left(u-\lambda_{j}\right)$. Define $\log ^{+} x=\max \{0, \log x\}$ for $x \geqq 0$. Yuzvinskii's result is that

$$
h\left(\alpha_{R_{1} / \mathrm{j}}\right)=\log \left|c_{m}\right|+\sum_{j=1}^{m} \log ^{+}\left|\lambda_{j}\right| .
$$

Jensen's formula [A, Thm. 5.3.1] implies

$$
\log ^{+}|\lambda|=\int_{T} \log \left|e^{2 \pi i t}-\lambda\right| d t .
$$

An elementary proof of this is given in [Yn]. Hence

$$
\begin{aligned}
h\left(\alpha_{R_{1} / f}\right) & =\log \left|c_{m}\right|+\int_{\mathbb{T}} \log \left|\prod_{j=1}^{m}\left(e^{2 \pi i t}-\lambda\right)\right| d t \\
& =\int_{\mathbb{T}} \log \left|f\left(e^{2 \pi i t}\right)\right| d t=\log \mathrm{M}(f) .
\end{aligned}
$$

We now turn to the case $d \geqq 2$. We will first obtain the lower bound $h\left(\alpha_{R_{d} / \mathfrak{f}}\right) \geqq \log \mathrm{M}(f)$ by use of the separated set definition of entropy (cf. Appendix A), approximation of $X_{R_{d} /}$ from inside by 1-dimensional solenoids, a 1-dimensional approximation to $\mathrm{M}(f)$ due to Boyd [By1] and Lawton [Lw], and the $d=1$ case already proved. We then establish the upper bound $h\left(\alpha_{R_{d} / f}\right) \leqq \log M(f)$ by use of the volume definition of entropy and a Riemann sum approximation to the integral defining $M(f)$.

Let us first introduce an explicit metric $\rho$ on $\mathbb{T} \mathbb{Z}^{a}$. For $s, t \in \mathbb{T}$, put $|s-t|=$ $\operatorname{dist}(s+\mathbb{Z}, t+\mathbb{Z})$. For $x, y \in \mathbb{T} \mathbb{Z}^{d}$ put

$$
\rho(x, y)=\sum_{\mathbf{j} \in \mathbb{Z}^{d}} 2^{-|\mathbf{j}|}|x(\mathbf{j})-y(\mathbf{j})|,
$$

where $|\mathbf{j}|=\left|\left(j_{1}, \ldots, j_{d}\right)\right|=\max \left\{\left|j_{1}\right|, \ldots,\left|j_{d}\right|\right\}$. The following lemma relates coordinatewise distances to this metric.

Lemma 3.2. If $\varepsilon>0$, there is $a \delta=\delta_{d}(\varepsilon)$ and an integer $b=b_{d}(\varepsilon)>0$ such that if $\rho(x, y)>\delta$, then there is $a \mathbf{j} \in \mathbb{Z}$ with $|\mathbf{j}|<b$ and $|x(\mathbf{j})-y(\mathbf{j})|>\varepsilon$.

Proof. Let $\sum_{\mathbf{j} \in \mathbb{Z}^{d}} 2^{-|\mathbf{j}|}=K<\infty$. Choose $b_{d}(\varepsilon)$ so that $\sum_{|\mathrm{j} j| \geqq b_{d}(\varepsilon)} 2^{-|\mathbf{j}|}<\varepsilon$, and put $\delta_{d}(\varepsilon)=(K+1) \varepsilon$. If $|x(\mathbf{j})-y(\mathbf{j})| \leqq \varepsilon$ for all $|\mathbf{j}|<b_{d}(\varepsilon)$, then

$$
\rho(x, y) \leqq \varepsilon+\sum_{|\mathbf{j}|<b_{d}(\varepsilon)} 2^{-|\mathbf{j}|} \varepsilon \leqq(K+1) \varepsilon=\delta_{d}(\varepsilon) .
$$


We will use this result as follows. If $\varepsilon>0$, then Lemma 3.2 shows that there exists $b>0$ and $\delta>0$ such that, for every rectangle $Q$ with sides of length $l_{j}$, and for every $(Q, \delta)$-separated set $F \subset \mathbb{T} \mathbb{Z}^{d}$ (cf. Appendix A), there exists a smaller rectangle $Q^{\prime}$ in $Q$ with sides of length $l_{j}-2 b$ such that $F$ is $\left(Q^{\prime}, \varepsilon\right)$-separated in the maximum distance over the coordinates of $Q^{\prime}$. Thus, in computing $h_{\text {sep }}(\alpha)$, we can use the maximum distance.

Suppose that $f \in R_{d}$ has the form $f(\mathbf{u})=\sum c_{\mathbf{j}} \mathbf{u}^{\mathbf{j}}$. For $\mathbf{r} \in \mathbb{Z}^{d}$ define a polynomial of one variable $f_{\mathrm{r}} \in R_{1}$ by

$$
f_{\mathbf{r}}(u)=f\left(u^{\mathrm{r}}\right)=f\left(u^{r_{1}}, \ldots, u^{r_{d}}\right)=\sum_{\mathbf{j} \in \mathbb{Z}^{d}} c_{j} u^{\mathrm{r} \cdot \mathbf{j}} .
$$

The values of $f_{\mathrm{r}}$ on $\mathbb{S}^{1}$ form a 1 -dimensional slice through the values of $f$ on $\mathbb{S}^{d}$. As these slices become more uniformly distributed, one would expect $M\left(f_{r}\right)$ to approximate $M(f)$. Boyd [By1] gave a precise formulation of this idea, and verified it for some special cases. Lawton $[\mathrm{Lw}]$ supplied an ingenious general proof for it. Put

$$
q(\mathbf{r})=\min \{|\mathbf{m}|: \mathbf{m} \neq \mathbf{0}, \mathbf{m} \cdot \mathbf{r}=0\} .
$$

Proposition 3.3 [Lawton]. For every $f \in R_{d}$ we have

$$
\lim _{q(\mathbf{r}) \rightarrow \infty} M\left(f_{\mathbf{r}}\right)=M(f) .
$$

To explain the reason for this, let $\mu_{\mathrm{r}}$ be Haar measure on the 1-dimensional subgroup of $\mathbb{T}^{d}$ obtained by projecting the line in $\mathbb{R}^{d}$ through $\mathbf{r}$. Then

$$
\mathrm{M}\left(f_{\mathrm{r}}\right)=\exp \left[\int_{\mathbb{J}^{d}} \log \left|f\left(e^{2 \pi i t}\right)\right| d \mu_{\mathrm{r}}(\mathbf{t})\right] .
$$

Now $\mu_{\mathbf{r}}$ converges weakly to Haar measure on $\mathbb{T}^{d}$ as $q(\mathbf{r}) \rightarrow \infty$. Hence if $f\left(e^{2 \pi i t}\right) \neq 0$ for $\mathbf{t} \in \mathbb{T}^{d}$, it is immediate that $\mathrm{M}\left(f_{\mathbf{r}}\right) \rightarrow \mathrm{M}(f)$. Possible zeros of $f\left(e^{2 \pi i t}\right)$ introduce logarithmic singularities in the integrand. Lawton handles this problem by obtaining an estimate on the measure of the set where $\left|f\left(e^{2 \pi i t}\right)\right|$ is small that depends only on the number of non-zero coefficients, and by employing the fact that $f_{\mathbf{r}}$ has the same number of non-zero coefficients if $q(\mathbf{r})$ is sufficiently large.

We call $\mathbf{r} \in \mathbb{Z}^{d}$ primitive if the greatest common divisor of its entries is 1 .

Fix a primitive $\mathbf{r} \in \mathbb{Z}^{d}$, and define $\psi_{\mathbf{r}} ; \mathbb{T}^{\mathbb{Z}} \rightarrow \mathbb{J}^{\mathbb{Z}^{d}}$ by $\left(\psi_{\mathbf{r}} \bar{x}\right)(\mathbf{j})=\bar{x}(\mathbf{r} \cdot \mathbf{j})$ for $\bar{x} \in \mathbb{\mathbb { T }}^{\mathbb{Z}}$. Since $\mathbf{r}$ is primitive, it follows that $\psi_{\mathbf{r}}$ is injective. To simplify notation, let $(X, \alpha)$ $=\left(X_{R_{d} / \mathrm{f}}, \alpha_{R_{d} / \mathfrak{F}}\right)$ and $\left(\bar{X}_{\mathrm{r}}, \bar{\alpha}_{\mathrm{r}}\right)=\left(X_{R_{1} / f_{\mathrm{r}}}, \alpha_{R_{1} / \tilde{f}_{\mathrm{r}}}\right)$, where $\tilde{f}_{\mathrm{r}}$ is the ideal generated by $f_{\mathrm{r}}$ in $R_{1}$. We claim that $\psi_{\mathbf{r}}\left(\bar{X}_{\mathbf{r}}\right) \subset X$. For if $\bar{x} \in \bar{X}_{\mathbf{r}}$ and $\mathbf{r} \cdot \mathbf{n}=n$, then

by (3-4).

$$
\sum_{\mathbf{j} \in \mathbb{Z}^{d}} c_{\mathbf{j}}\left(\psi_{\mathbf{r}} \bar{x}\right)(\mathbf{n}+\mathbf{j})=\sum_{\mathbf{j} \in \mathbb{Z}^{d}} c_{\mathbf{j}} \bar{x}(n+\mathbf{r} \cdot \mathbf{j})=0
$$

For $n \geqq 1$ let $\mathbf{r}_{n}=\left(1, n, n^{2}, \ldots, n^{d-1}\right)$. Note that $\mathbf{r}_{n}$ is primitive, and that $q\left(\mathbf{r}_{n}\right)=n \rightarrow \infty$ with $n$ (cf. (3-5) and [By1, p. 118]). Let

$$
Q_{n, m}=\{0,1, \ldots, n-1\}^{d-1} \times\{0,1, \ldots, m-1\} .
$$


Then the map $\mathbf{j} \mapsto \mathbf{r}_{n} \cdot \mathbf{j}$ gives a bijection of $Q_{n, m}$ to $\left\{0,1, \ldots,\left|Q_{n, m}\right|-1\right\}$. Let $b_{1}(\varepsilon)$ be determined by Lemma 3.2, and set $\tilde{Q}_{n, m}=\left\{b_{1}(\varepsilon), \ldots,\left|Q_{n, m}\right|-1-b_{1}(\varepsilon)\right\}$. Fix $\theta>0$. Let $\delta_{1}(\varepsilon)$ be determined by Lemma 3.2, so that $\delta_{1}(\varepsilon) \rightarrow 0$ as $\varepsilon \rightarrow 0$. By the definition of $h_{\text {sep }}\left(\bar{\alpha}_{\mathbf{r}_{\mathrm{n}}}\right.$ ) (cf. Appendix A), there exists an $\varepsilon>0$ such that, for all sufficiently large $n$, there exists an $m_{0}(\varepsilon, \theta, n) \geqq n$ such that if $m>m_{0}$ there exists a $\left(\tilde{Q}_{n, m}, \delta_{1}(\varepsilon)\right)$-separated set $\bar{F} \subset \bar{X}_{\mathbf{r}_{n}}$ such that

$$
|\vec{F}|>\exp \left[\left|\tilde{Q}_{n, m}\right|\left(h_{\text {sep }}\left(\bar{\alpha}_{\mathbf{r}_{n}}\right)-\theta\right)\right] .
$$

Suppose that $\bar{x}$ and $\bar{y}$ are distinct points in $\bar{F}$, and let $x=\psi_{\mathbf{r}_{n}}(\bar{x}), y=\psi_{\mathbf{r}_{n}}(\bar{y})$. Then there is an $i \in \tilde{Q_{n, m}}$ with $\rho\left(\bar{\alpha}_{\mathbf{r}_{n}}^{i} \bar{x}, \bar{\alpha}_{\mathbf{r}_{n}}^{i} \tilde{y}\right)>\delta_{1}(\varepsilon)$, where $\bar{\alpha}_{\mathbf{r}_{n}}^{i}$ denotes the $i$ th power of $\bar{\alpha}_{\mathbf{r}_{n}}$. By Lemma 3.2, there exists a $j$ with $0 \leqq j<\left|Q_{n, m}\right|$ such that $|\bar{x}(j)-\bar{y}(j)|>\varepsilon$. There is a unique $\mathbf{k} \in Q_{n, m}$ with $\mathbf{r}_{n} \cdot \mathbf{k}=j$, so $|x(\mathbf{k})-y(\mathbf{k})|>\varepsilon$, and $\rho\left(\alpha^{\mathbf{k} x} x, \alpha^{\mathbf{k}} y\right)>\varepsilon$. Hence $\psi_{\mathrm{r}_{n}}(\vec{F}) \subset X$ is a $\left(Q_{n, m}, \varepsilon\right)$-separated set for $\alpha$ with the same cardinality as $\vec{F}$. Thus, for $m \geqq m_{0}(\varepsilon, n, \theta)$,

$$
\frac{1}{\left|Q_{n, m}\right|} \log s_{Q_{n, m}}(\varepsilon, \alpha) \geqq \frac{\left|\tilde{Q}_{n, m}\right|}{\left|Q_{n, m}\right|}\left(h\left(\bar{\alpha}_{r_{n}}\right)-\theta\right) .
$$

Now the girth $g\left(Q_{n, m}\right) \rightarrow \infty$ as $n \rightarrow \infty$, and $\left|\tilde{Q}_{n, m}\right| /\left|Q_{n, m}\right| \rightarrow 1$ as $n \rightarrow \infty$. Also, $h\left(\bar{x}_{r_{n}}\right)=\log M\left(f_{r_{n}}\right) \rightarrow \log \mathrm{M}(f)$ by the first part of the proof and Proposition 3.3. By taking the lim sup as $n$ tends to infinity we see that

$$
s(\varepsilon, \alpha) \geqq \log M(f)-\theta
$$

for every $\theta>0$. Hence $h(\alpha) \geqq \log M(f)$, completing the proof of the lower bound.

We now turn to the proof of the upper bound $h(\alpha) \leqq \log M(f)$. We first transform $f$ into a form more convenient for our analysis. If $f(\mathbf{u})=\sum c_{\mathbf{j}} \mathbf{u}^{\mathrm{j}}$ and $A \in G L(d, \mathbb{Z})$, let $f^{A}(\mathbf{u})=\sum c_{\mathrm{j}} \mathbf{u}^{4 \mathrm{j}}$. Then $\alpha_{R_{d} /\left\langle f^{A}\right\rangle}$ is conjugate to $\alpha_{R_{d} / f}^{A}$, where as in $\S 2$ the action $\alpha^{A}$ is defined by $\left(\alpha^{A}\right)^{\mathrm{n}}=\alpha^{A \mathrm{n}}$. Now $h\left(\alpha^{A}\right)=h(\alpha)$, and an easy calculation using the fact that $A$ preserves Haar measure on $\mathbb{T}^{d}$ shows that $\mathrm{M}\left(f^{A}\right)=\mathrm{M}(f)$. Furthermore, both the action and its entropy are unaffected by multiplying $f$ by a monomial.

Thus by applying an appropriate $A \in G L(d, \mathbb{Z})$ and multiplying by a monomial, we can arrange that $f$ has the form

$$
f\left(u_{1}, \ldots, u_{d}\right)=q u_{d}^{D}+f_{D-1}\left(u_{1}, \ldots, u_{d-1}\right) u_{d}^{D-1}+\ldots+f_{0}\left(u_{1}, \ldots, u_{d-1}\right),
$$

where $q \neq 0$ is integral, $f_{k} \in \mathbb{Z}\left[u_{1}, \ldots, u_{d-1}\right]$, and $f_{0} \neq 0$. If $f$ is in this form and $f=\sum c_{\mathbf{j}} \mathbf{u}^{\mathbf{j}}$, then there is a $p>0$ such that

$$
\left\{\mathbf{j} \in \mathbb{Z}^{\mathbf{d}}: c_{\mathbf{j}} \neq 0\right\} \subset\left(\{0, \ldots, p-1\}^{d-1} \times\{0, \ldots, D-1\}\right) \cup\left\{D \mathbf{e}_{\mathbf{d}}\right\} .
$$

A major difficulty with higher dimensional Markov shifts is the so-called extension problem, namely whether a collection $x_{\mathbf{j}}$ of values for $\mathbf{j}$ in a subset of $\mathbb{Z}^{d}$ can be extended to an allowed point in the Markov shift. In full generality this problem is undecidable, and even in this algebraic setting it is not trivial (for a discussion of this, see [KS2]). One use of the transformation of $f$ to the form (3-6) is that a special case of the extension problem can be readily solved. 
Lemma 3.4. Suppose that $f \in R_{d}$ has the form (3-6), and let $S$ denote $\mathbb{Z}^{d-1} \times\{0, \ldots$, $D-1\}$. Then the homomorphism $X_{R_{d} / \uparrow} \rightarrow \mathbb{T}^{S}$ given by coordinate restriction is surjective.

Proof. If $x(\mathbf{j}) \in \mathbb{T}$ is arbitrary for $\mathbf{j} \in S$, we must show that $x$ can be extended to a point of $X_{R_{d} / \mathfrak{f}}$. If $\overline{\mathbf{u}}=\left(u_{1}, \ldots, u_{d-1}\right)$, write $f_{k}(\overline{\mathbf{u}})=\sum_{\mathbf{i} \in \mathbb{Z}^{d-1}} c_{k}(\mathbf{i}) \overline{\mathbf{u}}$. By (3-6), any extension of $x$ to $\mathbb{Z}^{d-1} \times\{D\}$ must satisfy, for $\mathbf{i} \in \mathbb{Z}^{d-1}$,

$$
q x(\mathbf{i}, D)=-\sum_{k=0}^{D-1} \sum_{\mathbf{m} \in \mathbb{Z}^{d-1}} c_{k}(\mathbf{m}) x(\mathbf{i}+\mathbf{m}, k) .
$$

Hence such an extension is possible, and for every $\mathbf{i} \in \mathbb{Z}^{d-1}$ there are $q$ choices for $x(i, D)$. Repeating this procedure extends $x$ to $\mathbb{Z}^{d-1} \times\{0,1, \ldots\}$. To extend $x$ to $\mathbb{Z}^{d-1} \times\{-1\}$ we must find values $x(\mathbf{i},-1), \mathbf{i} \in \mathbb{Z}^{d-1}$, so that

$$
\sum_{\mathbf{m} \in \mathbb{Z}^{d-1}} c_{0}(\mathbf{m}) x(\mathbf{i}+\mathbf{m},-1)=-\sum_{k=1}^{D-1} \sum_{\mathbf{m} \in \mathbb{Z}^{d-1}} c_{k}(\mathbf{m}) x(\mathbf{i}+\mathbf{m}, k-1)-q x(\mathbf{i}, D-1) .
$$

Since $R_{d}$ is an integral domain and $f_{0} \neq 0$, the homomorphism $\hat{R}_{d-1} \rightarrow \hat{R}_{d-1}$ dual to multiplication by $f_{0}$ on $R_{d}$ is surjective, so the extension exists. Repeated application of this argument extends $x$ to a point in $X_{R_{d} / \mathrm{f}}$.

Let $Y_{n}$ denote the set of points in $X_{R_{d} / /}$ which have period $n$ in each of the first $d-1$ coordinates. Then $Y_{n}$ is a closed $\alpha_{R_{d} / f}$-invariant subgroup. The following remarks will show that there is a constant $a>0$ independent of $n$ such that, for every $m>0$, the projection of $Y_{n}$ onto the coordinates

$$
\{0, \ldots, n-a m-1\}^{d-1} \times\{0, \ldots, m-1\}
$$

coincides with the projection of $X_{R_{d} / f}$ onto these coordinates. This justifies our replacement of $X_{R_{d} / f}$ by $Y_{n}$, and $\alpha_{R_{d} / f}$ by an automorphism $A$ of the finitedimensional torus $Y_{n}$ induced by the shift in the last coordinate.

Let $n$ be a positive integer, which should be considered very large compared to $p$. Put $Q_{/ n}=(\mathbb{Z} / n \mathbb{Z})^{d-1}, Q_{\mid n, D}=Q_{/ n} \times\{0, \ldots, D-1\}$, and put $\mathbb{T}_{n}=\mathbb{T}^{Q_{r n}}$, $\mathbb{T}_{n, D}=\pi Q_{\text {in.. }}$. If we identify $Q_{\mid n, D}$ with

$$
\{0, \ldots, n-1\}^{d-1} \times\{0, \ldots, D-1\} \subset \mathbb{Z}^{d},
$$

then Lemma 3.4 shows that the homomorphism $\varphi: X_{R_{d} / \varsubsetneqq} \rightarrow \mathbb{T}_{n, D}$ given by $\varphi(x)=\left.x\right|_{Q_{i n, D}}$ is surjective. Thus if $v=v_{n, D}$ is normalized Haar measure on $\mathbb{T}_{n, D}$, then $\varphi^{*}(\mu)=v$.

We shall assume from now on that $f \in R_{d}$ has the form (3-6), and that $p$ is given by (3-7). To simplify notation, we shall also assume that $q=1$ in (3-6). The changes needed for $|q|>1$ are indicated at the end of the proof.

There is a homomorphism $A: \mathbb{T}_{n, D} \rightarrow \mathbb{T}_{n, D}$ given by

$$
(A y)(\mathbf{i}, k)= \begin{cases}y(\mathbf{i}, k+1) & \text { if } 0 \leqq k \leqq D-2, \\ -\sum_{j=0}^{D-1} \sum_{\mathbf{m} \in\{0, \ldots, p-1\}^{d-1}} c_{j}(\mathbf{m}) y(\mathbf{i}+\mathbf{m}, j) & \text { if } k=D-1 .\end{cases}
$$


The relationship between $A$ and $\alpha$ is that the orbit of a point $y \in \mathbb{T}_{n, D}$ under $A$ and extension of $y$ to $X_{R_{d} / f}$ given by Lemma 3.4 must agree on the interior of a rectangle in the following sense. Let

$$
\tilde{Q_{n, m}}=\{0, \ldots, n-1-p m\}^{d-1} \times\{0, \ldots, m-1\},
$$

and suppose $(\mathbf{i}, k) \in \tilde{Q}_{n, m}$. If $y \in \mathbb{T}_{n, D}$, and $x$ is any point in $X_{R_{d} / f}$ with $\varphi(x)=y$, then $x(\mathbf{i}, k)=\left(A^{k} y\right)(\mathbf{i} \bmod n, 0)$. This observation is the basis of our proof for the upper bound.

Now fix an $\varepsilon_{1}>0$. Using the proof of Lemma 3.2 , let $\varepsilon=\left(2^{d}+1\right)^{-1} \varepsilon_{1}$, and $b=b_{d}(\varepsilon)$. If

then

$$
\bar{Q}_{n, m}=\{b, \ldots, n-1-p m-b\}^{d-1} \times\{b, \ldots, m-1-b\},
$$

$$
\left\{x \in X_{R_{d} / \digamma}:|x(\mathbf{j})|<\varepsilon, \mathbf{j} \in \tilde{Q}_{n, m}\right\} \subset \bigcap_{\mathbf{j} \in \bar{Q}_{n, m}} \alpha^{-\mathbf{j}} B\left(\varepsilon_{1}\right)=D_{\bar{Q}_{n, m}}\left(\alpha, \varepsilon_{1}\right) .
$$

For $y \in \mathbb{T}_{n, D}$, put $\|y\|_{\infty}=\max \left\{|y(\mathbf{j})|: \mathbf{j} \in Q_{n, D}\right\}$. By our observation from the previous paragraph, we have

$$
\varphi^{-1}\left\{y \in \mathbb{T}_{n, D}:\left\|A^{j} y\right\|_{\infty}<\varepsilon, 0 \leqq j<m\right\} \subset\left\{x \in X_{R_{d} / f}:|x(\mathbf{j})|<\varepsilon, \mathbf{j} \in \tilde{Q}_{n, m}\right\} .
$$

Thus to obtain an upper bound for $-\frac{1}{\left|\bar{Q}_{n, m}\right|} \log \mu\left(D_{\bar{Q}_{n, m}}\left(\alpha, \varepsilon_{1}\right)\right)$, it suffices to obtain one for

$$
-\frac{1}{\left|\bar{Q}_{n, m}\right|} \log v\left\{y \in \mathbb{T}_{n, D}:\left\|A^{j} y\right\|_{\infty}<\varepsilon, 0 \leqq j<m\right\} .
$$

Fix a sequence $m(n) \rightarrow \infty$ so that $m(n) / \log n \rightarrow \infty$ and $m(n) / n \rightarrow 0$ as $n \rightarrow \infty$. Then $g\left(\bar{Q}_{n, m(n)}\right) \rightarrow \infty$, and $\left|\bar{Q}_{n, m(n)}\right| /\left|Q_{(/ n, m(n)}\right| \rightarrow 1$, so in (3-10) we can replace $\bar{Q}_{n, m}$ with $Q_{j, m(n)}$.

We shall estimate the measure in (3-10) as follows. If $\mathbb{C}_{n}=\mathbb{C}_{1 n}$, then $A$ induces a linear map on $\mathbb{C}_{n}^{D}$. We will decompose $\mathbb{C}_{n}^{D}$ into an orthogonal direct sum of $D$ dimensional $A$-invariant subspaces indexed by $Q_{/ n}$. By adding up the volume decrease from the intersection in (3-10) over these subspaces we obtain a Riemann sum approximation to $\log M(f)$, from which the upper bound will follow.

Use the same symbol $A$ for the linear map $A: \mathbb{C}_{n}^{D} \rightarrow \mathbb{C}_{n}^{D}$ induced by the homomorphism $A$ of $\mathbb{T}_{n, D}$. Let $\|\cdot\|_{\infty}$ be the sup norm on $\mathbb{C}_{n}^{D}$, and let $v_{c}$ be Haar measure on $\mathbb{C}_{n}^{D}$ normalized so the unit cube has measure 1 . Then

$$
v\left\{y \in \mathbb{T}_{n, D}:\left\|A^{j} y\right\|_{\infty}<\varepsilon, 0 \leqq j<m\right\}=\left(v_{\mathrm{c}}\left\{z \in \mathbb{C}_{n}^{D}:\left\|A^{j} z\right\|_{\infty}<\varepsilon, 0 \leqq j<m\right\}\right)^{1 / 2},
$$

where the square root is necessary since passing to complex vector spaces squares volumes.

For $1 \leqq j \leqq d$ let $P_{j}$ act on $\mathbb{C}_{n}$ by $\left(P_{j} z\right)(\mathbf{i})=z\left(\mathbf{i}+\mathbf{e}_{j} \bmod n\right)$, and put $\mathbf{P}=\left(P_{1}, \ldots, P_{d-1}\right)$. Then the matrix of $A$ with respect to the standard basis on 
$\mathbb{C}_{n}^{D}$ is

$$
A=\left[\begin{array}{ccccc}
0 & I & 0 & \cdots & 0 \\
0 & 0 & I & \cdots & 0 \\
\vdots & \vdots & \vdots & \ddots & \vdots \\
0 & 0 & 0 & \cdots & I \\
-f_{0}(\mathbf{P}) & -f_{1}(\mathbf{P}) & -f_{2}(\mathbf{P}) & \cdots & -f_{D-1}(\mathbf{P})
\end{array}\right]
$$

Fix a primitive $n$th root of unity $\omega=e^{2 \pi i / n}$. For $\mathbf{k} \in Q_{\text {, }}$ define a vector $v_{\mathbf{k}} \in \mathbb{C}_{n}$ by $v_{\mathbf{k}}(\mathbf{j})=\omega^{\mathbf{j}} \cdot \mathbf{k} / \sqrt{\left.\mid Q_{\mid n}\right\}}$ for $\mathbf{j} \in Q_{/ n}$. The set $\left\{v_{\mathbf{k}}: \mathbf{k} \in Q_{i n}\right\}$ forms an orthonormal basis for $\mathbb{C}_{n}$. Also, $P_{j}\left(v_{\mathrm{k}}\right)=\omega^{k_{j}} \cdot v_{\mathrm{k}}$, so the $v_{\mathrm{k}}$ are simultaneous eigenvectors for the $P_{j}$.

Let $W_{\mathbf{k}}=\left(\mathbb{C} v_{\mathbf{k}}\right)^{D}$, equipped with the euclidean metric. Then $\mathbb{C}_{n}^{D}$ is the orthogonal direct sum of the $W_{\mathbf{k}}$. Furthermore, each $W_{\mathbf{k}}$ is an $A$-invariant $D$-dimensional complex subspace of $\mathbb{C}_{n}^{D}$, and the matrix of $A$ with respect to the standard basis on $W_{\mathbf{k}}$ is

$$
A_{\mathbf{k}}=\left.A\right|_{W_{\mathbf{k}}}=\left[\begin{array}{ccccc}
0 & 1 & 0 & \cdots & 0 \\
0 & 0 & 1 & \cdots & 0 \\
\vdots & \vdots & \vdots & \ddots & \vdots \\
0 & 0 & 0 & \cdots & 1 \\
-f_{0}\left(\omega^{\mathbf{k}}\right) & -f_{1}\left(\omega^{\mathbf{k}}\right) & -f_{2}\left(\omega^{\mathbf{k}}\right) & \cdots & -f_{D-1}\left(\omega^{\mathbf{k}}\right)
\end{array}\right] \text {, }
$$

where $\omega^{\mathbf{k}}=\left(\omega^{k_{1}}, \ldots, \omega^{k_{d-1}}\right)$ for $\mathbf{k} \in Q_{/ n}$.

For $\mathbf{k} \in Q_{/ n}$ let $v_{\mathbf{k}}$ be Haar measure on $W_{\mathbf{k}}$, normalized so the unit cube has measure 1 . Since the $v_{\mathbf{k}}$ are orthonormal, the normalizations agree so that $v_{\mathbb{C}}$ is the product of the $v_{\mathbf{k}}$. It is here that orthogonality of the $W_{\mathbf{k}}$ plays an essential role. Denote the unit ball in $W_{\mathbf{k}}$ by $B_{\mathbf{k}}$, and put $B_{*}=\oplus_{\mathbf{k}} B_{\mathbf{k}}$. Let $B_{\infty}(\varepsilon)$ be the $\varepsilon$-ball in $\mathbb{C}_{n}^{D}$ for $\|\cdot\|_{\infty}$. Since each coordinate of $v_{\mathbf{k}}$ has modulus $n^{-(d-1) / 2}$, and there are $n^{d-1}$ vectors, it follows that $B_{\infty}(\varepsilon) \supset \varepsilon n^{-(d-1)} B_{*}$. Hence

$$
\begin{aligned}
\left\{z \in \mathbb{C}_{n}^{D}:\left\|A^{j} z\right\|_{\infty}<\varepsilon, 0 \leqq j<m(n)\right\} & =\bigcap_{j=0}^{m(n)-1} A^{-j} B_{\infty}(\varepsilon) \\
& \supset \varepsilon n^{-(d-1)} \bigoplus_{\mathbf{k} \in Q_{n}}\left(\bigcap_{j=0}^{m(n)-1} A_{\mathbf{k}}^{-j} B_{\mathbf{k}}\right),
\end{aligned}
$$

so by (3-11),

$$
\begin{aligned}
& -\frac{1}{\left|Q_{n, m(n)}\right|} \log v\left\{y \in \mathbb{T}_{n, D}:\left\|A^{j} y\right\|_{\infty}<\varepsilon, 0 \leqq j<m(n)\right\} \\
& \quad \leqq-\frac{1}{\left|Q_{n, m(n)}\right|} \frac{1}{2} \log v_{\mathbb{C}}\left(\varepsilon n^{-(d-1)} \bigoplus_{\mathbf{k} \in Q_{i n}}\left(\bigcap_{j=0}^{m(n)-1} A_{\mathbf{k}}^{-j} B_{\mathbf{k}}\right)\right) \\
& \quad=\frac{\log \varepsilon^{-1}+(d-1) D\left|Q_{\mid n}\right| \log n}{2 m(n)\left|Q_{\mid n}\right|}+\frac{1}{2\left|Q_{\mid n}\right|} \sum_{\mathbf{k} \in Q_{i n}}-\frac{1}{m(n)} \log v_{\mathbf{k}}\left(\bigcap_{j=0}^{m(n)-1} A_{\mathbf{k}}^{-j} B_{\mathbf{k}}\right) \\
& \quad=O\left(\frac{\log n}{m(n)}\right)+\frac{1}{2\left|Q_{\mid n}\right|} \sum_{\mathbf{k} \in Q_{\mid n}} b_{m(n)}\left(A_{\mathbf{k}}\right),
\end{aligned}
$$


where the summands of the last line are defined by the summands in the previous line.

The last line of (3-12) contains a Riemann sum approximation. In order to prove this converges to the integral defining $\log M(f)$, we require the following uniformity result about the entropy of linear maps on $\mathbb{C}^{D}$. In what follows, if $T \in \mathbb{C}^{D \times D}$, then $h(T)$ denotes the Bowen entropy of $T$ (cf. $[\mathrm{Bw}]$ or $[\mathrm{W}, \mathrm{Ch} .7,8]$ ). The unit cube in $\mathbb{C}^{D}$ is the usual fundamental domain for the lattice $(\mathbb{Z}+i \mathbb{Z})^{D}$.

Lemma 3.5. Let $B$ be the unit ball in $\mathbb{C}^{D}$ for the euclidean norm, and $\mu$ be Haar measure on $\mathbb{C}^{D}$ normalized so the unit cube has measure 1 . For $T \in \mathbb{C}^{D \times D}$ put

$$
\begin{aligned}
b_{m}(T) & =-\frac{1}{m} \log \mu\left(\bigcap_{j=0}^{m-1} T^{-j} B\right), \\
h(T) & =\sum_{k=1}^{D} \log { }^{+}\left|\lambda_{k}\right|^{2}=2 \int_{\tau} \log \left|\chi_{T}\left(e^{2 \pi i t}\right)\right| d t,
\end{aligned}
$$

where $T$ has eigenvalues $\lambda_{k}$ counted with multiplicity and characteristic polynomial $\chi_{T}$. Then

(i) $h(T)$ and each $b_{m}(T)$ are continuous in $T$.

(ii) $b_{m}(T) \rightarrow h(T)$ uniformly on compact subsets of $\mathbb{C}^{D \times D}$.

Proof. (i) Fix $T \in \mathbb{C}^{D \times D}$ and $m \geqq 1$. To prove continuity of $b_{m}$ at $T$, let $\varepsilon>0$. Choose $\theta<1$ so $(2 D / m) \log \theta^{-1}<\varepsilon$. There is a neighborhood $\mathscr{U}$ of $T$ such that, if $\tilde{T} \in \mathscr{U}$, then $\left\|T^{j}-\tilde{T}^{j}\right\|<1-\theta$ for $0 \leqq j<m$. Hence

$$
\theta\left(\bigcap_{j=0}^{m-1} T^{-j} B\right)=\bigcap_{j=0}^{m-1} T^{-j}(\theta B) \subset \bigcap_{j=0}^{m-1} \tilde{T}^{-j} B .
$$

Since contracting a set by a factor of $\theta$ multiplies its measure by $\theta^{2 D}$, we obtain, by taking $-(1 / m) \log \mu(\cdot)$, that

$$
\varepsilon+b_{m}(T)>\frac{2 D \log \theta^{-1}}{m}+b_{m}(T) \geqq b_{m}(\tilde{T}) \quad(\tilde{T} \in \mathscr{U}) .
$$

The assumption $\left\|T^{j}-\tilde{T}^{j}\right\|<1-\theta$ is symmetric in $T$ and $\tilde{T}$, implying $\varepsilon+b_{m}(\tilde{T}) \geqq b_{m}(T)$ for $\tilde{T} \in \mathscr{U}$ as well, completing the proof of continuity of $b_{m}$ at $T$.

Now $b_{m}(T) \rightarrow h(T)$ for every $T \in \mathbb{C}^{D \times D}[\mathrm{~W}$, Thm. 8.14], so continuity of $h$ follows from that of the $b_{m}$ and part (ii).

(ii) To prove that $b_{m}(T) \rightarrow h(T)$ uniformly on compact subsets of $\mathbb{C}^{D \times D}$, it suffices to show that, for fixed $T$ and $\varepsilon>0$, there is an $m_{0}$ and a neighborhood $\mathscr{U}$ of $T$ such that, for every $\tilde{T} \in \mathscr{U}$ and every $m \geqq m_{0}$, we have $\left|b_{m}(\tilde{T})-h(\tilde{T})\right|<\varepsilon$. We do this by applying spectral theory to $T$ to show that the estimates from [W, Thm. 8.14] can be made uniform.

Let the eigenvalues of $T$ without multiplicity be $\left\{\zeta_{k}:-r \leqq k \leqq s\right\}$, indexed so $\left|\zeta_{k}\right| \leqq 1$ for $-r \leqq k \leqq 0$ and $\left|\zeta_{k}\right|>1$ for $1 \leqq k \leqq s$. Denote the multiplicity of $\zeta_{k}$ by $N_{k}$. Let $\gamma>0$, whose value will be determined by several conditions and estimates. The first condition is that the circles $C_{k}$ around $\zeta_{k}$ of radius $\gamma$ be disjoint. Put

$$
E_{k}=E_{k}(T)=\frac{1}{2 \pi i} \int_{C_{k}}(\zeta I-T)^{-1} d \zeta .
$$


According to [Kt, $\S \mathrm{I} .5 .3], E_{k}$ is the projection onto the generalized eigenspace $V_{k}$ of $T$ for $\zeta_{k}$ along the sum of the other generalized eigenspaces. Hence $\operatorname{dim}_{\mathbb{C}} V_{k}=N_{k}$. Let $\|\cdot\|$ be the euclidean norm on $\mathbb{C}^{D}$. Adapt this norm on each $V_{k}$ to the dynamics of $T$ by putting, for $y \in V_{k}$,

$$
\|y\|_{k}=\sum_{n=0}^{\infty} \frac{\left\|T^{n} y\right\|}{\left(\left|\zeta_{k}\right|+\gamma\right)^{k}} .
$$

The spectral radius formula shows that this series converges geometrically. If $T_{k}$ denotes $\left.T\right|_{V_{k}}$, then we also have that $\left\|T_{k} y\right\|_{k}<\left(\left|\zeta_{k}\right|+\gamma\right)\|y\|_{k}$ for $0 \neq y \in V_{k}$. For subsets $K$ and $L$ of $\mathbb{C}^{D}$, we say $K \Subset L$ if the closure of $K$ is contained in $L$. Let $B_{k}$ be the unit ball in $V_{k}$ in the norm $\|\cdot\|_{k}$. Then

$$
\left(\left|\zeta_{k}\right|+\gamma\right)^{-1} B_{k} \Subset T_{k}^{-1} B_{k} .
$$

Put $B_{*}=\bigoplus_{k=-r}^{s} B_{k}$, which is a bounded neighborhood of 0 . Hence there is a $\theta>1$ so that $\theta^{-1} B \Subset B_{*} \Subset \theta B$.

We now describe the effect on this situation of perturbing $T$ slightly to $\tilde{T}$. Let $E_{k}(\tilde{T})=\frac{1}{2 \pi i} \int_{c_{k}}(\zeta I-\tilde{T})^{-1} d \zeta$. Since the inverse of a matrix is a rational function of its entries, $E_{k}(\tilde{T})$ is analytic in $\tilde{T}$ for $\tilde{T}$ near $T$. In particular, there are exactly $N_{k}$ eigenvalues of $\tilde{T}$ within $C_{k}$. Let $\tilde{V}_{k}$ be the range of $E_{k}(\tilde{T})$. Since $E_{k}(\tilde{T})$ is close to $E_{k}(T)$, by $[\mathrm{Kt}, \S \mathrm{I} .4 .6]$ there exists $U_{k}(\tilde{T}) \in G L(D, \mathbb{C})$ such that

$$
E_{k}(\tilde{T})=U_{k}(\tilde{T})^{-1} E_{k}(T) U_{k}(\tilde{T}),
$$

$U_{k}(T)=I$, and $U_{k}(\tilde{T})$ is analytic for $\tilde{T}$ close to $T$. Carry over the $\|\cdot\|_{k}$ norm on $V_{k}$ to $\tilde{V}_{k}$ using $U_{k}(\tilde{T})$. Let $\tilde{B}_{k}$ denote the unit ball in $\tilde{V}_{k}$ under this norm, and put $\tilde{B}_{*}=\bigoplus_{k} \tilde{B}_{k}$. Let $\tilde{T}_{k}=\tilde{T}_{\tilde{V}_{k}}$. By continuity we have that $\left(\left|\zeta_{k}\right|+\gamma\right)^{-1} \tilde{B}_{k} \Subset \tilde{T}_{k}^{-1} \tilde{B}_{k}$ and $\theta^{-1} B \Subset \tilde{B}_{*} \Subset \theta B$.

Let $\varepsilon>0$. We will first show that $b_{m}(\tilde{T})>h(\tilde{T})-\varepsilon$ for large enough $m$ and $\tilde{T}$ close enough to $T$. Clearly

Hence,

$$
\bigcap_{j=0}^{m-1} \tilde{T}^{-j} \tilde{B}_{*} \subset\left(\bigoplus_{k=-r}^{0} \tilde{B}_{k}\right) \oplus\left(\bigoplus_{k=1}^{s} \tilde{T}_{k}^{-(m-1)} \tilde{B}_{k}\right) .
$$

$$
-\frac{1}{m} \log \mu\left(\bigcap_{j=0}^{m-1} \tilde{T}^{-j} \tilde{B}_{*}\right) \geqq-\frac{1}{m} \log \mu\left(\tilde{B}_{*}\right)+\frac{m-1}{m} \sum_{k=1}^{s} \log \left|\operatorname{det} \tilde{T}_{k}\right|^{2},
$$

the determinant being squared since we are working over complex rather than real vector spaces. For $k \geqq 1$ every eigenvalue of $\tilde{T}_{k}$ lies inside $C_{k}$, and therefore has modulus $>1$ provided that $\gamma$ is small enough. Since there are at most $D$ other eigenvalues of $\tilde{T}$, each having modulus $<1+\gamma$, we obtain that

$$
\sum_{k=1}^{s} \log \left|\operatorname{det} \tilde{T}_{k}\right|^{2} \geqq h(\tilde{T})-2 D \log (1+\gamma) .
$$


Since $\theta^{-1} B \subset \tilde{B}_{*}$, it follows that

$$
\theta^{-1} \bigcap_{j=0}^{m-1} \tilde{T}^{-j} B \subset \bigcap_{j=0}^{m-1} \tilde{T}^{-j} \tilde{B}_{*} .
$$

As in part (i) we get, by using (3-14) and (3-15), that

$$
\begin{aligned}
\frac{2 D \log \theta}{m}+b_{m}(\tilde{T}) & \geqq-\frac{1}{m} \log \mu\left(\bigcap_{j=0}^{m-1} \tilde{T}^{-j} \tilde{B}_{*}\right) \\
& \geqq\left(1-\frac{1}{m}\right) h(\tilde{T})-\frac{1}{m} \log \mu(\theta B)-2 D \log (1+\gamma) .
\end{aligned}
$$

Thus, for sufficiently small $\gamma$, it will follow that $b_{m}(\tilde{T})>h(\tilde{T})-\varepsilon$ for all sufficiently large $m$ and all $\tilde{T}$ sufficiently close to $T$.

We complete the proof of the lemma by obtaining the opposite inequality $b_{m}(\tilde{T})<h(\tilde{T})+\varepsilon$. By the above, $\left(\left|\zeta_{k}\right|+\gamma\right)^{-j}{B_{k}} \Subset \widetilde{T}_{k}^{-j} \tilde{B}_{k}$. Hence

$$
\bigcap_{i=0}^{m-1} \tilde{T}^{-j} \tilde{B}_{*} \supset\left(\bigoplus_{k=-r}^{0}(1+\gamma)^{-(m-1)} \tilde{B}_{k}\right) \oplus\left(\bigoplus_{k=1}^{s}\left(\left|\zeta_{k}\right|+\gamma\right)^{-(m-1)} \tilde{B}_{k}\right) \text {. }
$$

Thus

$$
\mu\left(\bigcap_{j=0}^{m-1} \tilde{T}^{-j} \tilde{B}_{*}\right) \geqq \mu\left(\tilde{B}_{*}\right)(1+\gamma)^{-2 D(m-1)} \prod_{k=1}^{s}\left(\left|\zeta_{k}\right|+\gamma\right)^{-2 N_{k}(m-1)} .
$$

Since $\tilde{B}_{*} \subset \theta B$, an argument as above shows that

$$
-\frac{1}{m} \log \mu\left(\bigcap_{j=0}^{m-1} \tilde{T}^{-j} \tilde{B}_{*}\right) \geqq-\frac{2 D \log \theta}{m}+b_{m}(\tilde{T}) .
$$

Applying $-(1 / m) \log \mu(\cdot)$ to (3-16) and using (3-17) shows that

$$
\begin{aligned}
b_{m}(\tilde{T})-\frac{2 D \log \theta}{m} \leqq & \frac{m-1}{m} \sum_{k=1}^{s} 2 N_{k} \log \left(\left|\zeta_{k}\right|+\gamma\right)-\frac{1}{m} \log \mu\left(\tilde{B}_{*}\right) \\
& +\frac{m-1}{m} 2 D \log (1+\gamma) \\
\leqq & \sum_{k=1}^{s} 2 N_{k} \log \left(\left|\zeta_{k}\right|+\gamma\right)-\frac{1}{m} \log \left(\theta^{-1} B\right)+2 D \log (1+\gamma) .
\end{aligned}
$$

Since $\tilde{T}$ has exactly $N_{k}$ eigenvalues within $\gamma$ of $\zeta_{k}$, all of modulus $>1$, and the remaining eigenvalues have modulus $<1+\gamma$, the sum $\sum_{k=1}^{s} 2 N_{k} \log \left(\left|\zeta_{k}\right|+\gamma\right)$ is arbitrarily close to $h(\tilde{T})$ provided $\gamma$ is small enough. Hence for $\gamma$ small enough, we will have $b_{m}(\tilde{T})<h(\tilde{T})+\varepsilon$ for all sufficiently large $m$ and all $\tilde{T}$ sufficiently close to $T$.

For $\mathbf{s} \in \mathbb{S}^{d-1}$ let

$$
A(\mathbf{s})=\left[\begin{array}{ccccc}
0 & 1 & 0 & \cdots & 0 \\
0 & 0 & 1 & \cdots & 0 \\
\vdots & \vdots & \vdots & \ddots & \vdots \\
0 & 0 & 0 & \cdots & 1 \\
-f_{0}(\mathbf{s}) & -f_{1}(\mathbf{s}) & -f_{2}(\mathbf{s}) & \cdots & -f_{D-1}(\mathbf{s})
\end{array}\right]
$$


the companion marix of its characteristic polynomial $f(\mathbf{s}, u)$. Then $A_{\mathbf{k}}=A\left(\omega_{\mathbf{k}}\right)$, and the sum in the last line of (3-12) is a Riemann sum approximating the integral.

$$
\frac{1}{2} \int_{\mathbb{J}^{d-1}} h\left(A\left(e^{2 \pi i t}\right)\right) d \mathbf{t}=\int_{\mathbb{T}^{d-1}} \int_{\mathbb{T}} \log \left|f\left(e^{2 \pi i t}, e^{2 \pi i s}\right)\right| d s d \mathbf{t}=\log \mathrm{M}(f) .
$$

To complete the proof of the theorem, let $\delta>0$. Since $\left\{A\left(e^{2 \pi i t}\right): t \in \mathbb{T}^{d-1}\right\}$ is compact in $\mathbb{C}^{D \times D}$, Lemma 3.5 and (3-18) imply that, for sufficiently large $n$,

$$
\begin{aligned}
\frac{1}{2\left|Q_{\mid n}\right|} & \sum_{k \in Q_{\mid / n}} b_{m(n)}\left(A_{k}\right)=\frac{1}{2\left|Q_{\mid n}\right|} \sum_{k \in Q_{j n}} b_{m(n)}\left(A\left(e^{2 \pi i \mathbf{k} / n}\right)\right) \\
& <\frac{1}{2} \int_{\delta^{d-1}} h\left(A\left(e^{2 \pi i \mathbf{t}}\right)\right) d \mathbf{t}+\delta=\log \mathrm{M}(f)+\delta .
\end{aligned}
$$

Using (A-4) and (3-11), it follows that

$$
s_{\bar{Q}_{n, m(n)}}\left(\varepsilon_{1}, \alpha\right) \leqq \frac{\left|Q_{n, m(n)}\right|}{\left|\bar{Q}_{n, m(n)}\right|}\left(o\left(\frac{\log n}{m(n)}\right)+\log \mathrm{M}(f)+\delta\right) .
$$

Since $m(n) / n \rightarrow 0$ we have that $\left|Q_{n, m(n)}\right| /\left|\bar{Q}_{n, m(n)}\right| \rightarrow 1$, and since $(\log n) / m(n) \rightarrow 0$, for large enough $n$ the right hand side of $(3-20)$ is less than $\log \mathrm{M}(f)+2 \delta$. Now $g\left(\bar{Q}_{n, m(n)}\right) \rightarrow \infty$ as $n \rightarrow \infty$, and Proposition A.2 implies that $s\left(4 \varepsilon_{1}, \alpha\right) \leqq \log M(f)$ $+2 \delta$. By letting $\varepsilon_{1} \rightarrow 0$ and then $\delta \rightarrow 0$ we conclude that $h(\alpha) \leqq \log M(f)$. This completes the proof of the upper bound.

Finally, we indicate the modifications necessary for the case where $|q|>1$. The estimate (3-19) for the linear map $q^{-1} A$ gives an upper bound of $\log \mathrm{M}\left(q^{-1} f\right)+\delta$. The $|q|$ possible choices in (3-8) contribute an additional term of $\log |q|$ to the volume decrease, just as in Yuzvinskii's calculation of entropy for solenoids [Yz2]. Since $\log M\left(q^{-1} f\right)=\log M(f)-\log |q|$, we again obtain an upper bound of $\log M(f)$ for $h(\alpha)$.

\section{§4. Entropy for general $\mathbb{Z}^{d}$ actions}

In this section we compute the entropy of an arbitrary $\mathbb{Z}^{\mathrm{d}}$-action $\alpha$ on a compact metrizable group $X$. We first show in Theorem 4.2 that if $\mathfrak{p}$ is a prime ideal in $R_{d}$ that is not principal, then $h\left(\alpha_{R_{d} / \mathfrak{p}}\right)=0$, while if $\mathfrak{p}=\langle f\rangle$ is principal, then $h\left(\alpha_{R_{d} / \mathfrak{p}}\right)=\log \mathrm{M}(f)$ by Theorem 3.1. If $X$ is abelian, and $(X, \alpha)$ has the descending chain condition, then $X$ has dual group $M$ that is a noetherian $R_{d}$-module. We show in Lemma 4.3 that $M$ contains a finite chain of submodules with successive quotients each isomorphic to $R_{d}$ modulo a prime ideal, so $h(\alpha)$ can be computed using the addition formula and Theorem 4.2. A general abelian $X$ is an inverse limit of groups with the descending chain condition, hence $h(\alpha)$ is the limit of entropies of actions whose value has already been computed. A general compact group is built from a totally disconnected part, a product of algebraically simple connected Lie groups, and an abelian part. In Theorem 4.5 we describe how these pieces fit together, show how to compute the entropy of each, and obtain $h(\alpha)$ as the sum of these entropies. 
Which real numbers can arise as $h(\alpha)$ ? By Theorem 3.1 all the numbers $\log M(f)$ for $f \in R_{d}$ occur, and our analysis will show that if $(X, \alpha)$ has the descending chain condition, then $h(\alpha)$ has this form. For general actions the problem is equivalent to another posed by Lehmer over fifty years ago, and still not solved. We show in Theorem 4.6 that the set of entropies of $\mathbb{Z}^{d}$-actions is either the countable set of logarithms of the Mahler measure of elements in $R_{d}$, or all of $[0, \infty]$, depending on the answer to Lehmer's problem. This generalizes Theorem 9.3 in [Ln3], where it is also shown that $\mathbb{\mathbb { Z }}$ either has no ergodic automorphisms of finite entropy, or has ergodic automorphisms of every finite positive entropy, again depending on the answer to Lehmer's problem (we have added the necessary "ergodic" assumption to this statement, which was inadvertently omitted from the last sentence in [Ln3, Thm. 9.3]).

We begin with a lemma needed a handle non-principal prime ideals.

Lemma 4.1. Suppose $\mathfrak{p} \neq 0$ is a non-principal prime ideal in $R_{d}$. For every $f \neq 0$ in $\mathfrak{p}$ there is a $g \neq 0$ in $\mathfrak{p}$ with no factor in common with $f$.

Proof. Suppose $0 \neq f \in \mathfrak{p}$, and let $f_{1}, \ldots, f_{r}$ be the distinct irreducible factors of $f$. Put $p_{j}=\left\langle f_{j}\right\rangle$, which is prime since $R_{d}$ is a unique factorization domain. Suppose every $0 \neq g \in \mathfrak{p}$ has a factor in common with $f$. Then $\mathfrak{p} \subset \mathfrak{p}_{1} \cup \ldots \cup \mathfrak{p}_{r}$, so by [AM, Prop. 1.11], $\boldsymbol{p} \subset \mathfrak{p}_{j}$ for some $j$. Since any principal prime ideal is minimal, this implies that $\mathfrak{p}=\mathfrak{p}_{j}$, i.e. that $\mathfrak{p}$ is principal. This contradiction proves the lemma.

Recall our convention that $M(0)=\infty$.

Theorem 4.2. Suppose $\mathfrak{p}$ is a prime ideal in $R_{d}$. Then

$$
h\left(\alpha_{R_{d} / \mathfrak{p}}\right)= \begin{cases}\log \mathrm{M}(f) & \text { if } \mathfrak{p}=\langle f\rangle, \\ 0 & \text { if } \mathfrak{p} \text { is not principal } .\end{cases}
$$

Proof. If $\mathfrak{p}=\langle f\rangle$, the result follows from Theorem 3.1.

Suppose that $\mathfrak{p}$ is not principal. By Lemma 4.1, there are $f, g \neq 0$ in $\mathfrak{p}$ with no common factor. Let $\mathfrak{i}=\langle f\rangle, \mathfrak{g}=\langle g\rangle, \mathfrak{a}=\mathfrak{j}+\mathfrak{g}=\langle f, g\rangle, R=R_{d}$. Let $\varphi: R / \mathfrak{f} \rightarrow R / \mathfrak{f}$ be multiplication by $g$. Since $f$ and $g$ have no common factor, $\varphi$ is injective. The image of $\varphi$ is

$$
\mathfrak{g}(R / \mathfrak{f})=\mathfrak{g} / \mathfrak{f} \mathfrak{g}=\mathfrak{g} / \mathfrak{f} \cap \mathfrak{g} \cong(\mathfrak{f}+\mathfrak{g}) / \mathfrak{f}=\mathfrak{a} / \mathfrak{f} .
$$

Hence we obtain the exact sequence

$$
0 \rightarrow R / \mathfrak{i} \stackrel{\varphi}{\rightarrow} R / \mathfrak{\uparrow} \rightarrow R / \mathfrak{a} \rightarrow 0 .
$$

Since $h\left(\alpha_{R / f}\right)=\log \mathrm{M}(f)<\infty$, the addition formula yields that $h\left(\alpha_{R / \mathfrak{a}}\right)=0$. Now $\mathfrak{a} \subset \mathfrak{p}$, and the projection $R / \mathfrak{a} \rightarrow R / \mathfrak{p}$ has dual the inclusion $X_{R / \mathfrak{p}} \subset X_{R / \mathfrak{a}}$ which respects the actions. Hence $h\left(\alpha_{R / \mathfrak{p}}\right)=0$.

For an action on a compact abelian group obeying the descending chain condition, the above results are sufficient to compute entropy.

Lemma 4.3. Suppose $M$ is a noetherian $R_{d}$-module. Then there is a chain of $R_{d^{-}}$ modules

$$
0=M_{0} \subset M_{1} \subset \ldots \subset M_{r-1} \subset M_{r}=M
$$


such that $M_{j} / M_{j-1} \cong R_{d} / \mathfrak{p}_{j}(1 \leqq j \leqq r)$, where each $\mathfrak{p}_{j}$ is a prime ideal. Hence

$$
h\left(\alpha_{M}\right)=\sum_{j=1}^{r} h\left(\alpha_{R_{d} / p_{j}}\right)
$$

where each summand is computed according to (4-1).

Proof. The existence of the chain $\left\{M_{j}\right\}$ is proved in $[\mathrm{Lg}, \mathrm{VI}, \mathrm{Cor} .4 .8]$. Use the addition formula repeatedly to obtain the formula for $h\left(\alpha_{M}\right)$.

Note that $\left(X_{M}, \alpha_{M}\right)$ satisfies the descending chain condition since $M$ is noetherian, and our proof shows that $h\left(\alpha_{M}\right)=\log M(g)$, where $g$ is the product of the generators of the principal prime ideals occurring in the $\mathfrak{p}_{j}$ (by convention, $g=1$ if none of the $\mathfrak{p}_{j}$ is principal).

Theorem 4.4. Suppose that $M$ is an arbitrary countable $R_{d}$-module. Then there is an increasing chain $\left\{M_{j}\right\}$ of noetherian submodules with union $M$. Thus $h\left(\alpha_{M}\right)=\lim _{j \rightarrow \infty}$ $h\left(\alpha_{M_{j}}\right)$, where each $h\left(\alpha_{M_{j}}\right)$ is computed by Lemma 4.3 .

Proof. The existence of the $M_{j}$ is routine. Since $X_{M_{j}} \cong X_{M} / M_{j}^{\perp}$, and $M_{j}^{\perp} \searrow\{1\}$, the partitions into cosets of $M_{j}^{\perp}$ converge to the partition into points, so $h\left(\alpha_{M_{j}}\right) \rightarrow(\alpha)$.

Theorem 4.5. Let $\alpha$ be a $\mathbb{Z}^{d}$-action on a compact metrizable group $X$. If $X^{0}$ is the connected component of the identity and $Z$ is the center of $X^{0}$, then

$$
h(\alpha)=h\left(\alpha_{X / X^{0}}\right)+h\left(\alpha_{X^{0} / Z}\right)+h\left(\alpha_{Z}\right) .
$$

The first summand is either the logarithm of an integer or $\infty$, the second is 0 or $\infty$, and the third is computed by Theorem 4.4 .

Proof. First observe that $X^{0}$ and $Z$ are both $\alpha$-invariant, so (4-2) follows from the addition formula.

Put $Y=X / X^{0}$, a totally disconnected compact metrizable group. There is a sequence $Y_{n}$ of compact open normal subgroups of $Y$ that decrease to $\{1\}$. For a closed normal subgroup $H$ of $Y$, let $\xi(H)$ denote the measurable partition of $Y$ into left cosets of $H$. Yuzvinskii $[\mathrm{Yz} 1, \S 2.1]$ has shown that $\xi\left(\bigcap_{n=1}^{\infty} H_{n}\right)=\bigvee_{n=1}^{\infty} \xi\left(H_{n}\right)$, and that if $H \subset K$, then $H_{\mu}(\xi(H) \mid \xi(K))=\log |K / H|$.

Let $P=\left\{\mathbf{j} \in \mathbb{Z}^{d}: \mathbf{j}<0\right\}$, where $<$ is lexicographic order, and put $P_{0}=P \cup\{\mathbf{0}\}$. For a measurable partition $\xi$ put $\xi_{P}=V_{\mathbf{j} \in P} \alpha^{\mathbf{j}} \xi$. By [C, Thm. 2.2], $h_{\mu}(\alpha, \xi)=H_{\mu}\left(\xi_{P_{0}} \mid \xi_{P}\right)$. Thus

$$
\begin{aligned}
h_{\mu}\left(\alpha, \xi\left(Y_{n}\right)\right) & =H_{\mu}\left(\xi\left(Y_{n}\right)_{P_{0}} \mid \xi\left(Y_{n}\right)_{P}\right)=H_{\mu}\left(\xi\left(\bigcap_{\mathrm{j} \in P_{0}} \alpha^{\mathrm{j}} Y_{n}\right) \mid \xi\left(\bigcap_{\mathbf{j} \in P} \alpha^{\mathrm{j}} Y_{n}\right)\right) \\
& =\log \left|\bigcap_{\mathrm{j} \in P} \alpha \mathrm{j} Y_{n} / \bigcap_{\mathrm{j} \in P_{0}} \alpha^{\mathrm{j}} Y_{n}\right|,
\end{aligned}
$$

which is the logarithm of an integer or $\infty$. Since $h\left(\alpha_{Y}\right)=\lim _{n \rightarrow \infty} h_{\mu}\left(\alpha, \xi\left(Y_{n}\right)\right)$, the same holds for $h\left(\alpha_{Y}\right)$.

Next consider $C=X^{0} / Z$, which is connected. By $[\mathrm{Yz} 1, \S 4.1], X^{0} / Z$, is centerless, and is a direct product $\prod_{i \in I} L_{i}$ of algebraically simple connected Lie groups. 
Furthermore, if $\mathbf{n} \in \mathbb{Z}^{d}$ and $i \in I$, then $\alpha_{C}^{\mathbf{n}}\left(L_{i}\right)=L_{j}$ for some $j \in I$, and this defines an action of $\mathbb{Z}^{d}$ on $I$. Since entropy adds over direct products, we may assume as in the proof of the addition formula that $\mathbb{Z}^{d}$ acts transitively on $I$. Fix $i_{0} \in I$, and let $L=L_{i_{0}}$. If there is an $\mathbf{n} \in \mathbb{Z} \backslash\{\mathbf{0}\}$ with $\alpha_{C}^{\mathbf{n}}(L)=L$, then $\alpha_{C}^{\mathbf{n}}$ is a direct product of automorphisms of simple Lie groups, and therefore has entropy 0 [Yz1, §4.2]. If $d=1$, this shows that $h\left(\alpha_{C}\right)=0$, while if $d>1$, then $h\left(\alpha_{C}\right)=0$ since some element of the action has finite entropy [C, Thm. 2.3]. If $\mathbb{Z}^{d}$ acts freely on $I$, then $\alpha_{C}$ is isomorphic to the shift on $L^{\mathbb{Z}^{a}}$, and since $L$ is infinite we have $h\left(\alpha_{C}\right)=\infty$.

Finally, since $Z$ is abelian, $Z=X_{M}$ for some countable $R_{d}$-module $M$, so $h\left(\alpha_{Z}\right)$ is computed via Theorem 4.4 .

The proof of the previous theorem shows that if $(X, \alpha)$ satisfies the descending chain condition, then $h(\alpha)=\log M(f)$ for some $f \in R_{d}$. Whether any other real numbers occur as entropies is closely related to the following problem.

Lehmer's problem. Is 1 a cluster point of $\{M(f): f \in \mathbb{Z}[u]\}$ ?

By Proposition 3.3, this is equivalent to determining whether 1 is a cluster point of $\left\{\mathrm{M}(f): f \in R_{d}\right\}$. This question was posed by Lehmer [Lh] over fifty years ago in connection with a method to factor large integers, and remains unsolved. The smallest known value $>1$ for a Mahler measure is that of a tenth degree polynomial discovered by Lehmer himself, whose measure is about 1.176280821 . The problem has been the subject of a number of investigations. Boyd's survey [By2] gives an excellent account of this and related problems, and supplies supporting evidence for his fascinating conjecture that $\bigcup_{d=1}^{\infty}\left\{\mathrm{M}(f): f \in R_{d}\right\}$ is a closed subset of $\mathbb{R}$, which would immediately give a negative answer to Lehmer's problem.

Theorem 4.6. The set of possible entropies of $\mathbb{Z}^{d}$-actions on compact groups is $[0, \infty]$ if the answer to Lehmer's problem is "yes", or is the countable set $\{\log M(f)$ : $\left.f \in R_{d}\right\}$ if the answer is "no".

Proof. If $\log M(f)$ can be arbitrarily small, then by taking countable products any positive real number can be obtained as the entropy of a $\mathbb{Z}^{d}$-action. If not, then in the approximation in Theorem 4.4 of $(X, \alpha)$ by quotients obeying the descending chain condition, each stage contributes either 0 or $\log M(f)$ for some $f \in R_{d}$ to the entropy. If $h(\alpha)<\infty$, then there can be only finitely many positive contributions, and $h(\alpha)$ has the form $\log \mathrm{M}(g)$ for some $g \in R_{d}$.

\section{§5. Examples and remarks}

This section contains examples of the foregoing results as well as some miscellaneous remarks about them.

Example 5.1. Let $f(u, v)=1+u+v \in R_{2}$, and $\mathfrak{j}=\langle f\rangle$. Then $X_{R_{2} / \mathfrak{f}}$ can be described as the set of all $x \in \mathbb{T}^{2}$ such that $x_{i, j}+x_{i+1, j}+x_{i, j+1}=0$ for all $i, j \in \mathbb{Z}$. This condition shows that every horizontal line of coordinates determines the next 
line above it by a simple additive rule, reminiscent of the evolution of a cellular automation, except here the alphabet is $\pi$ instead of a finite set. Smyth [Sm2] has explicitly computed $\mathrm{M}(f)$ with the result that

$$
h\left(\alpha_{R_{2} / \uparrow}\right)=\log \mathrm{M}(f)=\frac{3 \sqrt{3}}{4 \pi} \sum_{n=1}^{\infty}\left(\frac{n}{3}\right) \frac{1}{n^{2}}=\frac{3 \sqrt{3}}{4 \pi} L\left(2, \chi_{3}\right) \cong .3230659472,
$$

where $\left(\frac{n}{3}\right)$ is the Legendre symbol, $\chi_{3}(n)$ is the non-trivial character on $\mathbb{Z}_{/ 3}$, and $L(2, \chi)=\sum_{n=1}^{\infty} \chi(n) / n^{2}$ is the Dirichlet $L$-series.

This polynomial was a principal motivating example for this paper. Here $\alpha_{R_{2} / \gamma}$ is mixing [KS1, Thm. 11.2(4)], has a dense set of periodic points [KS1, Thm. 7.2], but is not expansive since the variety of $f$ contains the point $\left(e^{2 \pi i / 3}, e^{4 \pi i / 3}\right)$ in $\mathbb{S}^{2}[\mathrm{Sc} 2$, $\mathrm{Thm} .3 .8]$. We show in the next section that $\alpha_{R_{2} / \uparrow}$ has completely positive entropy, and thus is mixing of all orders. We conjecture (cf. Conjecture 6.8) that $\alpha_{R_{2} / f}$ is measure-theoretically isomorphic to a Bernoulli $\mathbb{Z}^{d}$-action.

Example 5.2. Let $\mathfrak{p}=\langle 2,1+u+v\rangle$, a maximal, non-principal prime ideal in $R_{2}$. Then $X_{R_{2} / p}$ is isomorphic to the set of $x \in \mathbb{Z}_{i 2}^{\mathbb{Z}^{2}}$ with $x_{i, j}+x_{i+1, j}+x_{i, j+1}=0$ for all $i, j \in \mathbb{Z}$. This action was studied by Ledrappier [Ld], who showed that it is mixing but not 2 -fold mixing. Since $\mathfrak{p}$ is prime but not principal, we have $h\left(\alpha_{R_{2} / \mathrm{p}}\right)=0$ by Theorem 3.1. This can also be seen directly by a familiar argument from cellular automata theory.

Example 5.3. Smyth [Sm2] has computed the measures of several polynomials in terms of the Dirichlet $L$-series of the odd character $\chi_{n}$ of $\mathbb{Z}_{1 n}$. A sampler of his calculations is given in Table 1 . In that table $\rho=(1+\sqrt{5}) / 2$ and $\chi_{5}(2)=i$.

Table 1. Values of entropy for some principal ideals

\begin{tabular}{ll}
\hline$f \in R_{d}$ & $h\left(\alpha_{R_{d} / 1}\right)$ \\
\hline$u+v$ & 0 \\
$u+v \pm 1$ & $\frac{3 \sqrt{3}}{4 \pi} L\left(2, \chi_{3}\right)$ \\
$u+v+w \pm 1$ & $\frac{7 \zeta(3)}{2 \pi^{2}}$ \\
$u+v \pm k$ & $\log |k|,|k| \geqq 2$ \\
$(u+v)^{2} \pm 2$ & $\frac{1}{2} \log 2+\frac{2}{\pi} L\left(2, \chi_{4}\right)$ \\
$(u+v)^{2} \pm 3$ & $\frac{2}{3} \log 3+\frac{\sqrt{3}}{\pi} L\left(2, \chi_{3}\right)$ \\
$(u+v)(u+v+1)-1$ & $\frac{2}{5} \log \rho+\frac{5^{3 / 4}}{4 \pi} \operatorname{Re}\left\{\left(\rho^{3 / 2}+i \rho^{-3 / 2}\right) L\left(2, \chi_{5}\right)\right\}$ \\
$u^{2}-v^{2}+u v+3 u-v+1$ & $2 \log \rho$ \\
\hline
\end{tabular}


Example 5.4. Results of Boyd and Smyth provide a complete characterization of those of $f \in R_{d}$ with $h\left(\alpha_{R_{d} / \mathfrak{f}}\right)=0$. An extended cyclotomic polynomial $g \in R_{d}$ is one of the form $g(\mathbf{u})=\mathbf{u}^{\mathbf{k}} \Phi\left(\mathbf{u}^{\mathrm{m}}\right)$, where $\Phi(u)$ is a cyclotomic polynomial in one variable, and $\mathbf{k}, \mathbf{m} \in \mathbb{Z}^{d}$. Using deep results of Schinzel, Boyd [By 1] proved that $M(f)=1$ if and only if $f$ is \pm 1 times a product of extended cyclotomic polynomials. Smyth [Sm1] found a simpler and more geometric proof.

Remark 5.5. If $f(\mathbf{u})=\Sigma_{\mathbf{j} \in \mathbb{Z}^{d}} c_{\mathbf{j}} \mathbf{u} \mathbf{j}$, let $\Sigma(f)=\left\{\mathbf{j} \in \mathbb{Z}^{d}: c_{\mathbf{j}} \neq 0\right\}$. A face $F$ of $\Sigma(f)$ is the intersection of $\Sigma(f)$ with a supporting hyperplane of its convex hull. Each face $F$ of $\Sigma(f)$ determines a polynomial $f_{F}(\mathbf{u})=\sum_{\mathbf{j} \in F} c_{\mathbf{j}} \mathbf{u}^{\mathbf{j}}$. Smyth [Sm1, Thm. 2] proved that $\mathrm{M}(f) \geqq \mathrm{M}\left(f_{F}\right)$ for every face $F$ of $\Sigma(f)$, which he used in his inductive approach to proving the result described in Example 5.4. Boyd [By2, $\$ 6]$ has exploited this to show, for example, that if $d=2$ and the convex hull of $\Sigma(f)$ has an odd number of sides, then $M(f) \geqq M(1+u+v) \cong 1.381356444$.

Smyth's inequality can be proven dynamically as follows. We may suppose that $f \in R_{d}$ has the form (3-6), and that $F=\Sigma(f) \cap\left(\mathbb{R}^{d-1} \times\{0\}\right)$. Then $f_{F}=f_{0} \in R_{d-1}$. If $x_{\mathbf{j}}$ is defined for $\mathbf{j} \in H=\mathbb{Z}^{d-1} \times\{0,1, \ldots\}$, and is the restriction of a point in $X_{R_{d} / f}$, then the possible extensions to $\mathbf{j} \in \mathbb{Z}^{d-1} \times\{-1\}$ are governed by (3-9). Every such extension can be modified by the addition of an arbitrary element from $X_{R_{d-1}\left\langle\left\langle f_{0}\right\rangle\right.}$, i.e. by an extension of a point whose jth coordinate is 0 for all $\mathbf{j} \in H$. From this it follows that $h\left(\alpha_{R_{d} / \mathfrak{f}}\right) \geqq h\left(\alpha_{R_{d-1} /\left\langle f_{0}\right\rangle}\right)$, and hence that $\mathrm{M}(f) \geqq \mathrm{M}\left(f_{0}\right)=\mathrm{M}\left(f_{F}\right)$.

Example 5.6. Let $A$ be an $n \times n$ matrix over $R=R_{d}$, and consider the $R$-module $R^{n} / A R^{n}$. A generalization of our main formula (3-1) is that

$$
h\left(\alpha_{R^{n} / A R^{n}}\right)=\log \mathrm{M}(\operatorname{det} A) .
$$

In order to prove (5-1), first suppose that $\operatorname{det} A=0$. Then $R^{n} / A R^{n}$ contains an $R$-torsion-free element $x$, so that $\alpha_{R^{n} / A R^{n}}$ has a factor $\alpha_{R x}$ of infinite entropy. Hence $h\left(\alpha_{R^{n} / A R^{n}}\right)=\infty=\log \mathrm{M}(0)$.

Thus we assume for the remainder of the proof that all matrices have non-zero determinant. We next show that if (5-1) holds for $A$ and for $B$, then it holds for $A B$. Consider the exact sequence

$$
0 \rightarrow A R^{n} / A\left(B R^{n}\right) \rightarrow R^{n} / A\left(B R^{n}\right) \rightarrow R^{n} / A K^{n} \rightarrow 0 .
$$

Since $\operatorname{det} A \neq 0$, the map $A$ is injective on $R^{n}$, so that

$$
A R^{n} / A\left(B R^{n}\right) \cong R^{n} / B R^{n} \text {. }
$$

Use of the addition formula then shows that

$$
\begin{aligned}
h\left(\alpha_{R^{n} / A B R^{n}}\right) & =h\left(\alpha_{R^{n} / A R^{n}}\right)+h\left(\alpha_{R^{n} / B R^{n}}\right) \\
& =\log \mathrm{M}(\operatorname{det} A)+\log \mathrm{M}(\operatorname{det} B)=\log \mathrm{M}(\operatorname{det} A B),
\end{aligned}
$$

establishing (5-1) for $A B$.

Computation of the echelon form for $A$, considered as a matrix over the field of fractions of $R$, shows that there is a non-zero $f \in R$ such that $f A$ is a product of 
elementary matrices over $R$, each having the form

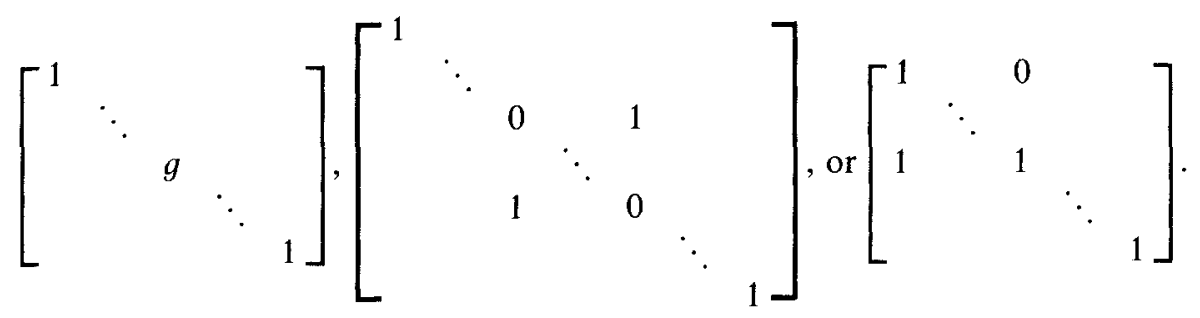

Now (5-1) easily holds for each of the elementary matrices by (3-1), and hence for their product $f A$. Thus

$$
h\left(\alpha_{R^{n} / f A R^{n}}\right)=\log \mathrm{M}(\operatorname{det}(f A))=n \log \mathrm{M}(f)+\log \mathrm{M}(\operatorname{det} A) .
$$

Putting $B=f I$ in (5-2) and using (3-1) shows that

$$
\begin{aligned}
h\left(\alpha_{R^{n} / f A R^{n}}\right) & =h\left(\alpha_{R^{n} / f R^{n}}\right)+h\left(\alpha_{R^{n} / A R^{n}}\right) \\
& =n \log \mathrm{M}(f)+h\left(\alpha_{R^{n} / A R^{n}}\right) .
\end{aligned}
$$

Since $\mathrm{M}(f)<\infty$, these show that $h\left(\alpha_{R^{n} / A R^{n}}\right)=\log \mathrm{M}(\operatorname{det} A)$.

Example 5.7. Suppose $f(u) \in \mathbb{Z}[u]$ and $g(v) \in \mathbb{Z}[v]$ are both monic with constant term \pm 1 . Put $a=\langle f(u), g(v)\rangle$. Then $X_{R_{2} / \mathfrak{a}} \cong \mathbb{J}^{m}$, where $m=(\operatorname{deg} f) \cdot(\operatorname{deg} g)$. Then $\alpha_{R_{2} / \mathrm{a}}$ acts by commuting toral automorphisms. For appropriate choices of $f$ and $g$ (e.g. $f(u)=u^{2}-u-1$ and $g(v)=v^{2}-2 v-1$ ), every element $\alpha_{R_{2} / \mathbf{a}}^{\mathbf{n}}$ for $\mathbf{n} \neq 0$ of this action is Bernoulli. However $h\left(\alpha_{R_{2} / a}\right)=0$ since some element of the action has finite entropy [C, Thm. 2.3].

Remark 5.8. If $f \in R_{d}$, and $e \geqq 1$, then $f \in R_{d+e}$ as well. However, $M(f)$ is independent of $e$, since the extra variables integrate to 1 . To see this dynamically, let $\alpha=\alpha_{R_{d} / R_{d} f}$. Then $\alpha_{R_{d+e} / R_{d+e} f}$ is isomorphic to an action $\bar{\alpha}$ of $\mathbb{Z}^{d} \times \mathbb{Z}^{e}$ on $X_{R_{d} / R_{d} f}^{\mathbb{Z}^{e}}$ defined by

$$
\left(\bar{\alpha}^{(\mathbf{m}, \mathbf{n})}\right) x(\mathbf{k})=\left(\alpha^{\mathbf{m}} x\right)(\mathbf{k}+\mathbf{n}) .
$$

Since the $\mathbb{Z}^{e}$ part of this action is just a group shift, it is easy to see from, say, the volume definition of entropy that $h(\bar{\alpha})=h(\alpha)$.

Remark 5.9. It is perhaps interesting to note that $\mathrm{M}(f)$ is actually the " $L$ " norm of $f$ on $\mathbb{S}^{d}$. From $[\mathrm{Rd}, \mathrm{Ex} .3 .5(d)]$ we have

$$
M(f)=\lim _{p \searrow 0}\|f\|_{L^{p}\left(S^{d}\right)} .
$$

\section{§6. Completely positive entropy and Berg's theorem}

Let $M$ be an $R_{d}$-module, and $\alpha_{M}$ be the corresponding $\mathbb{Z}^{d}$-action on $X_{M}$. In this section we identify the Pinsker $\sigma$-subalgebra of $\alpha_{M}$, and show that $\alpha_{M}$ has completely positive entropy if and only if all the prime ideals associated to $M$ are 
principal and non-cyclotomic. We investigate in more detail the quotients that arise in the various prime filtrations of $M$, and in particular show that the minimal prime ideals associated to $M$ must occur, and with the same multiplicity, in every prime filtration of $M$. An example, however, shows that the quotients in a prime filtration, by themselves, are not sufficient to decide whether $\alpha_{M}$ has completely positive entropy. One application is to prove the analogue of a theorem of Berg, which states that if $\alpha_{M}$ has finite entropy, then it has completely positive entropy if and only if Haar measure is the unique measure of maximal entropy.

If $\alpha$ is a measure-preserving $\mathbb{Z}^{d}$-action on $(X, \mu)$, then the Pinsker partition $\pi(\alpha)$ is the supremum of all finite measurable partitions $\xi$ with $h_{\mu}(\xi, \alpha)=0$. The action $\alpha$ is said to have completely positive entropy if $\pi(\alpha)$ is the trivial partition. This is equivalent to requiring $h_{\mu}(\xi, \alpha)>0$ for all non-trivial finite measurable partitions.

Throughout this section we shorten $R_{d}$ to $R$. If $M$ is an $R$-module, we will determine $\pi\left(\alpha_{M}\right)$ and give a criterion for $\alpha_{M}$ to have completely positive entropy.

A point $x \in X_{M}$ is said to be $\alpha_{M}$-periodic if $x$ has finite orbit under $\alpha_{M}$.

Proposition 6.1. If $M$ is a noetherian $R$-module, then the set of $\alpha_{M}$-periodic points is dense in $X_{M}$.

Proof. Since $M$ is noetherian, $\alpha_{M}$ satisfies the descending chain condition on closed subgroups. A fundamental result [KS1, Thm. 7.2] of Kitchens and Schmidt is that this implies the density of $\alpha_{M}$-periodic points.

If $H$ is a closed subgroup of $X$, let $\xi(H)$ denote the measurable partition of $X$ into cosets of $H$.

Proposition 6.2. Suppose $X$ is a compact metrizable abelian group, and $\alpha$ is a $\mathbb{Z}^{d}$ action on $X$ whose periodic points are dense. Then there is a closed $\alpha$-invariant subgroup $H$ of $X$ such that the Pinsker partition $\pi(\alpha)$ is the coset partition $\xi(H)$.

Proof. Our argument generalizes Rohlin's for toral automorphisms [Rh, \$4.1]. Let $A$ be a finite-index subgroup of $\mathbb{Z}^{d}$, and choose an integral matrix $A$ with $A\left(\mathbb{Z}^{d}\right)=\Lambda$. Then $x \in X$ has $\alpha$-stabilizer $\Lambda$ if and only if $x$ is fixed by the action $\alpha^{A}$ defined by $\left(\alpha^{A}\right)^{\mathrm{j}}=\alpha^{A \mathrm{j}}$. In this case, translation by $x$ commutes with $\alpha^{A}$, so $\pi\left(\alpha^{A}\right)$ is invariant under this translation. Now $\pi\left(\alpha^{A}\right)=\pi(\alpha)$, so $\pi(\alpha)$ is invariant under a dense set of translations, hence all translations. This implies by [Rh, \$3.2] or [Ln1] that there is a closed subgroup $H$ of $X$ such that $\pi(\alpha)=\xi(H)$. Since $\pi(\alpha)$ is $\alpha$ invariant, so is $H$.

Call an ideal in $R$ cyclotomic if it is the principal ideal generated by an extended cyclotomic polynomial as defined in Example 5.4.

Definition 6.3. A prime ideal in $R$ is null if it is either non-principal or cyclotomic. A prime ideal is positive if it is principal and not cyclotomic (this includes the 0 ideal). The set of null ideals is denoted by $\mathscr{N}$ and that of positive ideals by $\mathscr{P}$.

The terminology is suggested by Example 5.4, which shows that $h\left(\alpha_{R / p}\right)>0$ if and only if $\mathfrak{p}$ is positive. 
Definition 6.4. A prime ideal $p$ in $R$ is associated to the $R$-module $M$ if there is and element $m \in M$ whose annihilator is $\mathfrak{p}$. The set of prime ideals associated to $M$ is denoted by asc $(M)$.

Theorem 6.5. Let $M$ be a countable R-module. There is a unique submodule $N$ of $M$ such that the Pinsker partition $\pi\left(\alpha_{M}\right)$ is the coset partition $\xi\left(N^{\perp}\right)$. Every prime ideal associated to $N$ is null. Hence $\alpha_{M}$ has entropy 0 if and only if all prime ideals associated to $M$ are null, and $\alpha_{M}$ has completely positive entropy if and only if all prime ideals associated to $M$ are positive.

Proof. Consider the collection $\mathscr{C}$ of submodules $N_{0}$ of $M$ with $h\left(\alpha_{N_{0}}\right)=0$, partially ordered by inclusion. Then $\mathscr{C}$ is not empty since it contains the 0 submodule. If $\left\{N_{j}\right\}$ is a chain in $\mathscr{C}$, and $N_{\infty}=\bigcup_{j} N_{j}$, then $h\left(\alpha_{N_{x}}\right)=\lim _{j} h\left(\alpha_{N_{j}}\right)=0$, showing that $N_{\infty} \in \mathscr{C}$ is an upper bound for the chain. By Zorn's lemma $\mathscr{C}$ has a maximal element $N$. Let $N^{\prime} \in \mathscr{C}$. The addition map $N \times N^{\prime} \rightarrow N+N^{\prime}$ dualizes to show that $\alpha_{N+N^{\prime}}$ is a subsystem of $\alpha_{N \times N^{\prime}}$. Hence

$$
h\left(\alpha_{N+N^{\prime}}\right) \leqq h\left(\alpha_{N \times N^{\prime}}\right)=h\left(\alpha_{N} \times \alpha_{N^{\prime}}\right)=h\left(\alpha_{N}\right)+h\left(\alpha_{N^{\prime}}\right)=0,
$$

so $N+N^{\prime} \in \mathscr{C}$. Maximality of $N$ shows therefore that $N^{\prime} \subset N$, proving uniqueness of $N$.

By Proposition 6.2, there is a submodule $P$ of $M$ such that $\pi\left(\alpha_{M}\right)=\xi\left(P^{\perp}\right)$. Then $h\left(\alpha_{P}\right)=h\left(\alpha, X_{M} / P^{\perp}\right)=0$, so $P \subset N$. Conversely, $\xi\left(N^{\perp}\right) \leqq \pi\left(\alpha_{M}\right)$ since $h\left(\alpha_{N}\right)=h\left(\alpha, X_{M} / N^{\perp}\right)=0$, so $N \subset P$. This proves $\pi\left(\alpha_{M}\right)=\xi\left(N^{\perp}\right)$.

If $\mathfrak{p} \in \operatorname{asc}(N)$, there is an $n \in N$ with $\operatorname{ann}(n)=\mathfrak{p}$. Then $R n \cong R / \mathfrak{p}$, so

$$
h\left(\alpha_{R / \mathfrak{p}}\right)=h\left(\alpha_{R n}\right) \leqq h\left(\alpha_{N}\right)=0,
$$

proving $\mathfrak{p} \in \mathscr{N}$.

If $h\left(\alpha_{M}\right)=0$, then $\pi\left(\alpha_{M}\right)=\xi(\{1\})$, so $N=M$ and $\operatorname{asc}(M)=\operatorname{asc}(N) \subset \mathscr{N}$. If $\operatorname{asc}(M) \subset \mathcal{N}$, then every submodule of the form $R m(m \in M)$ is in $\mathscr{C}$. Since $\mathscr{C}$ is closed under module addition, it follows that $N=M$, so $h\left(\alpha_{N}\right)=0$.

Suppose $\alpha_{M}$ has completely positive entropy, and let $p \in \operatorname{asc}(M)$. Choose $m \in M$ with $\mathfrak{p}=\operatorname{ann}(m)$. Then $h\left(\alpha_{R / \mathfrak{p}}\right)=h\left(\alpha_{R m}\right)>0$ since $\alpha_{R m}$ is a non-trivial factor of $\alpha_{M}$. Hence $p \in \mathscr{P}$, proving asc $(M) \subset \mathscr{P}$. Conversely, suppose asc $(M) \subset \mathscr{P}$, and let $N$ be the submodule of $M$ given above. If $N \neq\{0\}$, then there is a $p \in \operatorname{asc}(N) \subset \operatorname{asc}(M) \subset \mathscr{P}$, while by the above $\operatorname{asc}(N) \subset \mathscr{N}$, which is disjoint from $\mathscr{P}$. This contradiction forces $N=\{0\}$, so $\pi\left(\alpha_{M}\right)=\xi\left(N^{\perp}\right)$ is trivial, proving $\alpha_{M}$ has completely positive entropy.

Corollary 6.6. Suppose that $X$ is a compact abelian group, that $\alpha$ is a $\mathbb{Z}^{d}$-action on $X$, and that $Y$ is an $\alpha$-invariant closed subgroup of $X$. If both $\alpha_{Y}$ and $\alpha_{X / Y}$ have completely positive entropy, then so does $\alpha$.

Proof. There are $R$-modules $N \subset M$ so that $X=X_{M}$ and $Y=X_{N}=X_{M} / N^{\perp}$. The hypotheses show that $\operatorname{asc}(N) \subset \mathscr{P}$ and asc $(M / N) \subset \mathscr{P}$. By [Mt, Lemma 7.F],

$$
\operatorname{asc}(M) \subset \operatorname{asc}(N) \cup \operatorname{asc}(M / N) \subset \mathscr{P},
$$

so $\alpha_{M}$ has completely positive entropy by Theorem 6.5 . 
Completely positive entropy implies very strong mixing conditions. A measure preserving $\mathbb{Z}^{d}$-action $\alpha$ on $(X, \mu)$ is called mixing of order $r$ if, for every collection of measurable sets $B_{0}, B_{1}, \ldots, B_{r}$ in $X$, we have

$$
\mu\left(B_{0} \cap \alpha^{\mathbf{k}_{1}} B_{1} \cap \ldots \alpha^{\mathbf{k}_{r}} B_{r}\right) \rightarrow \mu\left(B_{0}\right) \mu\left(B_{1}\right) \ldots \mu\left(B_{r}\right)
$$

as $\max \left\{\left|\mathbf{k}_{i}-\mathbf{k}_{j}\right|: i \neq j\right\} \rightarrow \infty$.

Corollary 6.7. Let $M$ be a countable R-module. If all the prime ideals associated to $M$ are positive, then $\alpha_{M}$ is mixing of all orders.

Proof. This is immediate from Theorem 6.5 and the result of Kaminski [ $\mathrm{Km}$, $\mathrm{Thm} .2]$ that completely positive entropy implies mixing of all orders.

Note in particular that if $\mathfrak{p} \in \mathscr{P}$, Then $M=R / p$ satisfies the hypotheses, so $\alpha_{R_{i}}$ is mixing of all orders, proving a claim made in Example 5.1. For further results on the mixing properties implied by complete positivity of entropy, the reader is referred to Kaminski's paper.

When $d=1$, ergodicity implies completely positive entropy, which in turn implies the automorphism is measurably isomorphic to a Bernoulli shift [Ln3]. Since Bernoulli $\mathbb{Z}^{d}$-actions must have completely positive entropy, the appropriate conjecture for general $d$ is the following.

Conjecture 6.8. If $\alpha_{M}$ has completely positive entropy, then it is measurably isomorphic to a Bernoulli $\mathbb{Z}^{d}$-action.

We now turn to a more detailed study of the filtrations used in Lemma 4.3 to compute $h\left(\alpha_{M}\right)$ for a noetherian $R$-module $M$.

A prime filtration $\left\{M_{j}\right\}$ of $M$ is a chain of $R$-submodules

$$
0=M_{0} \subset M_{1} \subset \ldots \subset M_{r-1} \subset M_{r}=M,
$$

where $M_{j} / M_{j-1} \cong R / \mathfrak{p}_{j}$ with $\mathfrak{p}_{j}$ a prime ideal of $R(1 \leqq j \leqq r)$. A prime ideal $\mathfrak{p}$ occurs in the filtration (6-1) if $\mathfrak{p}=\mathfrak{p}_{j}$ for some $j$, and occurs with multiplicity $r$ if exactly $r$ of the $\mathfrak{p}_{j}$ equal $\mathfrak{p}$. If $M$ is noetherian, then $M$ is a torsion $R$-module if and only if every prime ideal occurring in a filtration is non-zero. In this case the annihilating ideal $\mathfrak{a}=\{f \in R: f \cdot M=0\}$ of $M$ is non-zero.

Proposition 6.9. Let $M$ be a noetherian $R$-module, and $p$ be a prime ideal minimal over the annihilator of $M$. Then $\mathfrak{p}$ occurs in every prime filtration of $M$, and with the same multiplicity in each.

Proof. If $\mathfrak{p}$ is minimal over $\mathbf{a}=\operatorname{ann}(M)$, then $\mathfrak{p} \in \operatorname{asc}(M)$ [Mt, Thm. 7.D]. If $\left\{M_{j}\right\}$ is a prime filtration of $M$ with $M_{j} / M_{j-1} \cong R / \mathfrak{p}_{j}$, then $\operatorname{asc}(M) \subset\left\{\mathfrak{p}_{j}\right\}$ [Mt, Prop. 7.6], showing $p$ occurs at least once in every prime filtration of $M$.

Invariance of multiplicity is based on showing that any pair of prime filtrations of $M$ have a common refinement, and that the multiplicity of a minimal prime over $a$ is preserved under refinement.

Suppose we have two prime filtrations of $M$. By the Schreier refinement theorem for modules [ $\mathrm{Lg}, \mathrm{IV}, \S 4$, Thm. 4], these filtrations have refinements with the same number of terms such that the quotients are isomorphic in pairs. An 
application of Lemma 4.3 to each pair of isomorphic quotients shows we can further refine these filtrations to be prime.

Let $M_{i-1} \subset M_{i}$ be part of a prime filtration of $M$, with $M_{i} / M_{i-1} \cong R / q$. Suppose this inclusion is refined to

$$
M_{i-1}=N_{0} \subset N_{i} \subset \ldots \subset N_{p-1}=M_{i} .
$$

Then $N_{k} / N_{0} \cong \mathfrak{a}_{k} / \mathfrak{q}$ for an ideal $\mathfrak{a}_{k}$ in $R$. If $N_{k} / N_{k-1} \cong R / \mathfrak{q}_{k}$ with $\mathfrak{q}_{k}$ prime, then $\mathfrak{q}_{k}=\operatorname{ann}\left(N_{k} / N_{k-1}\right)$. We claim $\mathfrak{q}=\mathfrak{q}_{1}$, while $\mathfrak{q} \nsubseteq \mathfrak{q}_{k}$ for $2 \leqq k \leqq p$. Since $R / \mathfrak{q}$ is an integral domain, and $\mathfrak{a}_{1} / \mathfrak{q} \neq 0$, the annihilator of $\mathfrak{a}_{1} / \mathfrak{q}$, considered as an $R / \mathfrak{q}$-module, is 0 . This shows that $\mathfrak{q}_{1}=\mathfrak{q}$. Also, for $2 \leqq k \leqq p$ we have $\mathfrak{q}_{k}=\operatorname{ann}\left(N_{k} / N_{k-1}\right) \supset \mathfrak{a}_{k-1} \neq \mathfrak{q}$.

Suppose $\mathrm{p}$ is a minimal prime over a. If $M_{i} / M_{i-1} \cong R / \mathfrak{p}$, then for any refinement (6-2), only the first quotient $N_{1} / N_{0}$ is isomorphic to $R / p$, while the others are isomorphic to $R / \mathfrak{q}_{j}$ with $\mathfrak{q}_{j} \neq \mathfrak{p}$. If $M_{i} / M_{i-1} \cong R / \mathfrak{q}$ with $\mathfrak{q} \neq \mathfrak{p}$, then minimality of $p$ shows that no quotient in (6-2) is isomorphic to $R / \mathfrak{p}$. Hence the multiplicity of $p$ is invariant under refinement.

Proposition 6.10. Suppose $M$ is a noetherian $R$-module. Then every minimal associated prime ideal of $M$ occurs, and with the same multiplicity, in every prime filtration of $M$. In particular, if $M$ is torsion, then this applies to every principal prime ideal associated to $M$.

Proof. The minimal associated prime ideals of $M$ are exactly the minimal primes over $\operatorname{ann}(M)[\mathrm{Mt}$, p. 51], and so Proposition 6.9 implies the first statement.

If $M$ is torsion, then $\operatorname{ann}(M) \neq 0$, hence a principal prime ideal over $\operatorname{ann}(M)$ is automatically minimal, and Proposition 6.9 again applies.

Remark 6.11. Suppose $M$ is noetherian, and that $\left\{M_{i}\right\},\left\{M_{j}^{\prime}\right\}$ are prime filtrations of $M$ with quotients isomorphic to $R / \mathfrak{p}_{i}$ and $R / p_{j}^{\prime}$, respectively. Theorem 4.3 gives two ways to compute entropy, namely

$$
h\left(\alpha_{M}\right)=\sum_{i} h\left(\alpha_{R / \mathfrak{p}_{1}}\right)=\sum_{i} h\left(\alpha_{R / \mathfrak{p}_{j}}\right) .
$$

The previous proposition shows these are equal for trivial reasons. If $M$ is torsion, then positive terms in each sum correspond to principal prime ideals by Theorem 4.2, so by Proposition 6.10 the positive terms in the second sum are the same as those in the first after a possible rearrangement. If $M$ is not torsion, then 0 is a minimal associated prime ideal, and thus at least one term in each sum is $\infty . \square$

It is possible to tell from the quotients of a prime filtration of $M$ whether $\alpha_{M}$ has completely positive entropy? Unfortunately, the answer is "no," even when $M$ is torsion. We are grateful to Paul Smith for help with the following example.

Example 6.12. Let $d=2, R=R_{2}, f(u) \in \mathbb{Z}[u]$ be irreducible and non-cyclotomic, $g(v)=v-1$, and define ideals in $R$ by

$$
\begin{aligned}
& \mathfrak{p}_{1}=\langle f(u)\rangle, \quad \mathfrak{p}_{2}=\langle f(u), g(v)\rangle, \quad \mathfrak{a}_{1}=\left\langle f(u), g(v)^{2}\right\rangle, \\
& \mathfrak{b}_{1}=\left\langle f(u)^{2}, g(v)\right\rangle, \quad \mathbf{b}_{2}=\left\langle f(u)^{2}, f(u) g(v)\right\rangle .
\end{aligned}
$$


Let $M=R / \mathfrak{p}_{1}$ and $M^{\prime}=R / \mathfrak{b}_{2}$. Consider the filtrations

$$
\begin{aligned}
& 0 \subset \mathfrak{a}_{1} / \mathfrak{p}_{1} \subset \mathfrak{p}_{2} / \mathfrak{p}_{1} \subset R / \mathfrak{p}_{1}=M, \\
& 0 \subset \mathfrak{b}_{1} / \mathfrak{b}_{2} \subset \mathfrak{p}_{2} / \mathfrak{b}_{2} \subset R / \mathfrak{b}_{2}=M^{\prime} .
\end{aligned}
$$

It is elementary to check that the first quotients $a_{1} / p_{1}$ and $b_{1} / b_{2}$ in each filtration are isomorphic to $R / \mathfrak{p}_{1}$, while all other quotients are isomorphic to $R / \mathfrak{p}_{2}$. Since $\mathfrak{p}_{1}$ and $\mathfrak{p}_{2}$ are prime ideals, these are prime filtrations with term-wise isomorphic quotients. However, $\operatorname{asc}(M)=\left\{p_{1}\right\} \subset \mathscr{P}$ while $\operatorname{asc}\left(M^{\prime}\right)=\left\{p_{1}, p_{2}\right\} \nsubseteq \mathscr{P}$, so by Theorem $6.5 \alpha_{M}$ has completely positive entropy while $\alpha_{M}$, does not.

The following does give some relationship between $\alpha_{M}$ having completely positive entropy and the quotients of a fixed prime filtration of $M$.

Theorem 6.13. Suppose $M$ is a torsion noetherian $R$-module. If $\alpha_{M}$ has completely positive entropy, and $\left\{M_{j}\right\}$ is a prime filtration of $M$, then the prime ideals minimal with respect to occurring in $\left\{M_{j}\right\}$ must all be positive. Conversely, if $\left\{M_{j}\right\}$ is a prime filtration of $M$ in which only positive prime ideals occur, then $\alpha_{M}$ has completely positive entropy.

Proof. Suppose first that $M$ has completely positive entropy. Let $\left\{M_{j}\right\}$ be a prime filtration of $M$, with $M_{j} / M_{j-1} \cong R / \mathfrak{p}_{j}$. Since $0 \notin \operatorname{asc}(M) \subset \mathscr{P}_{,}$all the associated prime ideals of $M$ are minimal, hence occur in $\left\{M_{j}\right\}$. Now the minimal associated prime ideals of $M$ are exactly the minimal prime ideals over ann $(M)$, and every $\mathfrak{p}_{j}$ lies over $\operatorname{ann}(M)$. Thus the set of minimal elements in $\left\{\mathfrak{p}_{j}\right\}$ is just asc $(M)$, and these are all positive.

If $\left\{M_{j}\right\}$ is a prime filtration with $M_{j} / M_{j-1} \cong R / \mathfrak{p}_{j}$ and $\mathfrak{p}_{j} \in \mathscr{P}$ for all $j$, then $\operatorname{asc}(M) \subset\left\{\mathfrak{p}_{j}\right\} \subset \mathscr{P}$, so $\alpha_{M}$ has completely positive entropy.

We now turn to the question of unique ergodicity for $\alpha_{M}$. In $[\mathrm{Bg}]$, Berg showed that an ergodic automorphism of a compact group with finite entropy is uniquely ergodic, with Haar measure being the unique measure of maximal entropy. His proof uses Yuzvinskii's result that for group automorphisms ergodicity implies completely positive entropy, and then uses the latter condition. The appropriate version of Berg's theorem for general $d$ is as follows.

Theorem 6.14. Suppose that $M$ is an R-module and $h\left(\alpha_{M}\right)<\infty$. Then Haar measure on $X_{M}$ is the unique measure of maximal entropy if and only if $\alpha_{M}$ has completely positive entropy.

Proof. First suppose $\alpha_{M}$ has completely positive entropy. Using the theory of measure-preserving $\mathbb{Z}^{d}$-action developed by Conze [C], most of Berg's proof for unique ergodicity generalizes directly. The one exception is Lemma 2.3 of $[\mathrm{Bg}]$, whose proof depends on the total order of $\mathbb{Z}$. We will give a different proof that is valid for all $d$.

If $\xi$ is a measurable partition of a Lebesgue space $X$, and $\alpha$ is a measure-

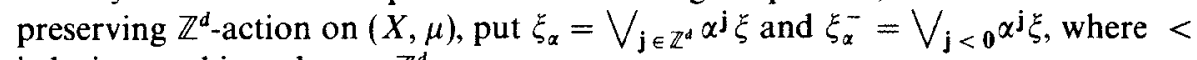
is lexicographic order on $\mathbb{Z}^{d}$. 
Lemma 6.15. Suppose $\xi$ and $\eta$ are measurable partitions of $X$ with finite entropy, and that $\alpha$ is a measure-preserving $\mathbb{Z}^{d}$-action on $(X, \mu)$. If

$$
h_{\mu}(\xi \vee \eta, \alpha)=h_{\mu}(\xi, \alpha)+h_{\mu}(\eta, \alpha),
$$

and $\alpha$ has completely positive entropy on $\xi_{\alpha}$, then the partitions $\xi_{\alpha}$ and $\eta_{\alpha}$ are independent.

Proof. Pinsker's formula [C, Eq. (19)] shows that (6-3) holds if and only if $H\left(\eta \mid \eta_{\alpha}^{-}\right)=H\left(\eta \mid \eta_{\alpha}^{-} \vee \xi_{\alpha}\right)$. This proves that if (6-3) holds, then for any $\zeta \leqq \xi$ we have $h(\zeta \vee \eta, \alpha)=h(\zeta, \alpha)+h(\eta, \alpha)$.

For $k \geqq 1$ let $\alpha^{k}$ denote the $\mathbb{Z}^{d}$-action defined by $\left(\alpha^{k}\right) \mathrm{j}=\alpha^{k \mathrm{j}}$. Put $Q_{k}=\{0,1, \ldots, k-1\}^{d}$. Then

$$
\begin{aligned}
h\left(\xi_{Q_{k}} \vee \eta_{Q_{k}}, \alpha^{k}\right) & =k^{d} h(\xi \vee \eta, \alpha)=k^{d} h(\xi, \alpha)+k^{d} h(\eta, \alpha) \\
& =h\left(\xi_{Q_{k}}, \alpha^{k}\right)+h\left(\eta_{Q_{k}}, \alpha^{k}\right) .
\end{aligned}
$$

Since $\xi \leqq \xi_{Q_{k}}$, our previous remark shows that

$$
h\left(\xi \vee\left(\eta_{Q_{k}}\right), \alpha^{k}\right)=h\left(\xi, \alpha^{k}\right)+h\left(\eta_{Q_{k}}, \alpha^{k}\right) .
$$

Hence $H\left(\xi \mid \xi_{\alpha^{k}}\right)=H\left(\xi \mid \xi_{\alpha^{k}}\left(\eta_{Q_{k}}\right)_{\alpha^{k}}\right)$. Since $\bigwedge_{k=1}^{\infty} \xi_{\alpha^{k}} \leqq \pi(\alpha)$, which is trivial by assumption, we have

$$
H(\xi \mid \eta) \geqq H\left(\xi \mid \xi_{\alpha^{k}}^{-} \vee \eta\right)=H\left(\xi \mid \xi_{\alpha^{k}}\right) \rightarrow H(\xi)
$$

as $k \rightarrow \infty$. Thus $\xi \perp \eta$. Now (6-3) implies via Pinsker's formula that

$$
h\left(\xi_{Q_{k}} \vee \eta_{Q_{k}}, \alpha\right)=h\left(\xi_{Q_{k}}, \alpha\right)+h\left(\eta_{Q_{k}}, \alpha\right)
$$

for every $k$, so our argument applies to show that $\xi_{Q_{k}} \perp \eta_{Q_{k}}$ for $k \geqq 1$. This proves that $\xi_{\alpha} \perp \eta_{\alpha}$.

Having estabilished Lemma 6.15 , the rest of the case when $\alpha_{M}$ has completely positive entropy is carried out exactly as in $[\mathrm{Bg}]$.

Finally, suppose $\alpha_{M}$ does not have completely positive entropy. By Theorem 6.5 there is a non-zero submodule $N \subset M$ with $h\left(\alpha_{N}\right)=0$. Now $X_{M / N}$ is a subgroup of $X_{M}$, and $\alpha_{M / N}$ is a subsystem of $\alpha_{M}$. The addition formula shows that $\alpha_{M / N}$ has the same entropy as $\alpha_{M}$. Thus Haar measure on $X_{M / N}$ is another $\alpha_{M}$-invariant measure of maximal entropy, so $\alpha_{M}$ is not uniquely ergodic.

\section{§7. Periodic points}

In this section we investigate the growth rate of the number of periodic points for a $\mathbb{Z}^{d}$-action, and prove that for expansive $\mathbb{Z}^{d}$-actions this growth rate coincides with the topological entropy.

If $\alpha$ is a $\mathbb{Z}^{d}$-action on a compact group $X$, and $\Lambda$ is a finite-index subgroup, or lattice, in $\mathbb{Z}^{d}$, then we put

$$
P_{\Lambda}(\alpha)=\left\{x \in X: \alpha^{\mathbf{n}} x=x \text { for all } \mathbf{n} \in \Lambda\right\},
$$

the subgroup of $\Lambda$-periodic points. Put $\|A\|=\operatorname{dist}(\mathbf{0}, A \backslash\{\mathbf{0}\})$, and denote the 
index of $\Lambda$ in $\mathbb{Z}^{d}$ by $\left|\mathbb{Z}^{d} / \Lambda\right|$. We will be concerned with the growth rates

$$
p^{-}(\alpha)=\liminf _{\|A\| \rightarrow \infty} \frac{1}{\left|\mathbb{Z}^{d} / \Lambda\right|} \log \left|P_{\Lambda}(\alpha)\right|, \quad p^{+}(\alpha)=\limsup _{\|\Lambda\| \rightarrow \infty} \frac{1}{\left|\mathbb{Z}^{d} / \Lambda\right|} \log \left|P_{\Lambda}(\alpha)\right|
$$

For arbitrary $\mathbb{Z}^{d}$-actions, little can be said about the relationship between $p^{ \pm}(\alpha)$ and $h(\alpha)$. For example, if $\alpha$ is the identity action on an infinite group, then $p^{ \pm}(\alpha)=\infty$, while $h(\alpha)=0$. An example of the reverse inequality occurs for $d=1$, $X=\hat{\mathbb{Q}}$, and $\alpha$ dual to multiplication by $3 / 2$. Then $\left|P_{A}(\alpha)\right|=1$ for all $\Lambda$ [LW, $\S 3$ ], so that $p^{ \pm}(\alpha)=0$, while $h(\alpha)=\log 3$ by Theorem 3.1. Moreover, the equality of $p^{-}(\alpha)$ and $p^{+}(\alpha)$ can involve delicate questions about the diophantine character of the logarithms of algebraic numbers (cf. $[\operatorname{Ln} 4, \S 4]$ ).

A $\mathbb{Z}^{d}$-action $\alpha$ on $X$ is called expansive if there is a neighborhood $U$ of the identity $0_{X}$ of $X$ such that $\bigcap_{\mathbf{n} \in \mathbb{Z}^{\alpha}} \boldsymbol{\alpha}^{\mathbf{n}} U=\left\{0_{X}\right\}$. Expansive actions have finite entropy since they possess finite generators. If $X$ is abelian, and $\alpha$ is an expansive $\mathbb{Z}^{d}$-action of $X$, then $\alpha$ has the descending chain condition [KS1, Thm. 5.2] so that $\hat{X}$ is noetherian, the subgroup $P_{A}(\alpha)$ is finite for every lattice $\Lambda \subset \mathbb{Z}^{d}$ (this follows directly from expansiveness, or see [Sc2, Cor. 3.8]), and the periodic points for $\alpha$ are dense in $X$ [KS1, Cor. 7.4].

If $\mathfrak{a}$ is an ideal in $R_{d}$, denote its complex variety by

$$
V(\mathfrak{a})=\left\{\mathbf{z}=\left(z_{1}, \ldots, z_{d}\right) \in \mathbb{C}^{d}: g(\mathbf{z})=0 \text { for every } g \in \mathfrak{a}\right\} .
$$

For $f \in R_{d}$, we put $V(f)=V(\langle f\rangle)$. Call a prime ideal $\mathfrak{p}$ expansive if

$$
V(p) \cap \mathbb{S}^{d}=\varnothing .
$$

If $M$ is an $R_{d}$-module, then $\alpha_{M}$ is expansive if and only if $M$ is noetherian and every prime ideal associated to $M$ is expansive [Sc2, Thm. 3.9]. In particular, suppose that $f \in R_{d}$ has irreducible factorization $f=g_{1}^{k_{1}} \times \ldots \times g_{r}^{k_{r}}$. Since $\operatorname{asc}\left(R_{d} /\langle f\rangle\right)=$ $\left\{\left\langle g_{1}\right\rangle, \ldots,\left\langle g_{r}\right\rangle\right\}$, and $V(f)=\bigcup_{j=1}^{r} V\left(g_{j}\right)$, it follows that $\alpha_{R_{d} /\langle f\rangle}$ is expansive if and only if $V(f) \cap \mathbb{S}^{d}=\varnothing$.

By use of the separated set definition of entropy (cf. Appendix A), it easily follows that for expansive actions $\alpha$ we have that

$$
p^{-}(\alpha) \leqq p^{+}(\alpha) \leqq h(\alpha) .
$$

The lack of equality in the above examples does not occur for expansive actions.

Theorem 7.1. Suppose that $M$ is an $R_{d}$-module, and that the $\mathbb{Z}^{d}$-action $\alpha_{M}$ is expansive on $X_{M}$. Then

$$
p^{-}\left(\alpha_{M}\right)=p^{+}\left(\alpha_{M}\right)=h\left(\alpha_{M}\right),
$$

so that

$$
\lim _{\|\Lambda\| \rightarrow \infty} \frac{1}{\left|\mathbb{Z}^{d} / \Lambda\right|} \log \left|P_{\Lambda}(\alpha)\right|=h\left(\alpha_{M}\right) .
$$

We shall prove Theorem 7.1 by first establishing some preliminary algebraic results, then handling the case when $M$ is a primary module, and finally using the primary decomposition theorem to show (7-2) for a general module. 
If $A \subset \mathbb{Z}^{d}$ is a lattice, let

$$
\mathfrak{b}_{\Lambda}=\left\langle\mathbf{u}^{\mathbf{n}}-1: \mathbf{n} \in A\right\rangle \subset R_{d} .
$$

If $\left\{\mathbf{n}_{1}, \ldots, \mathbf{n}_{d}\right\}$ is an integral basis for $\Lambda$, then $b_{A}$ is also the ideal generated by the finite set $\left\{\mathbf{u}^{\mathbf{n}_{j}}-1: 1 \leqq j \leqq d\right\}$.

Lemma 7.2. Suppose that $M$ is an $R_{d}$-module, and that $\Lambda$ is a lattice in $\mathbb{Z}^{d}$. Then the dual group of $P_{A}\left(\alpha_{M}\right)$ is isomorphic to $M / b_{A} M$. Hence

Proof. Duality shows that

$$
\left|P_{A}\left(\alpha_{M}\right)\right|=\left|M / \mathfrak{b}_{A} M\right| \text {. }
$$

Hence

$$
\operatorname{ker}\left(\alpha_{M}^{\mathbf{n}}-I\right)^{\perp}=\left(\mathbf{u}^{\mathbf{n}}-1\right) M
$$

$$
P_{A}\left(\alpha_{M}\right)^{\perp}=\left[\bigcap_{n \in A} \operatorname{ker}\left(\alpha_{M}^{n}-I\right)\right]^{\perp}=\sum_{n \in A}\left(u^{n}-1\right) M=b_{A} M
$$

so that

$$
P_{A}\left(\alpha_{M}\right)^{-} \cong M / P_{A}\left(\alpha_{M}\right)^{\perp}=M / \mathfrak{b}_{A} M
$$

Lemma 7.3. Suppose that $f \in R_{d}$, and that $f$ vanishes on $V\left(\mathfrak{b}_{A}\right)$. Then $f \in \mathfrak{b}_{A}$.

Proof. Pick a complete set $K \subset \mathbb{Z}^{d}$ of coset representatives for $\mathbb{Z}^{d} / \Lambda$. Note that $V\left(b_{A}\right)$ is a finite multiplicative subgroup of $S^{d}$. Each monomial $\mathbf{u}^{\mathrm{j}}$, restricted to $V\left(b_{A}\right)$, gives a group character of $V\left(b_{A}\right)$, and an elementary argument shows that we may identify the character group of $V\left(b_{A}\right)$ with

$$
\left\{\left.\mathbf{u}\right|_{V\left(\mathrm{~b}_{A}\right)}: \mathbf{j} \in K\right\} \cong V\left(\mathrm{~b}_{A}\right)^{\hat{x}} \text {. }
$$

Let $\mathbb{Z}\left[V\left(b_{A}\right)^{\hat{n}}\right]$ be the integral group ring of $V\left(b_{A}\right)$, and consider the restriction $\operatorname{map} \psi: R_{d} \rightarrow \mathbb{Z}\left[V\left(\mathrm{~b}_{A}\right)\right]$ given by

$$
\psi\left(\sum c_{\mathbf{j}} \mathbf{u}^{\mathbf{j}}\right)=\sum c_{\mathbf{j}}\left(\left.\mathbf{u}^{\mathrm{j}}\right|_{\nu\left(\mathbf{b}_{A}\right)}\right)
$$

Clearly $\mathfrak{b}_{A} \subset \operatorname{ker} \psi$.

Suppose that $f \in R_{d}$, and that $f$ vanishes on $V\left(\mathfrak{b}_{A}\right)$. If $f \notin \mathfrak{b}_{A}$, then $f \equiv g\left(\bmod \mathfrak{b}_{A}\right)$, where $g=\sum_{\mathbf{j} \in K} c_{\mathbf{j}} \mathbf{u}^{\mathbf{j}} \neq 0$. But then $0=\psi(f)=\psi(g)$ would give a non-trivial relation for the characters of $V\left(b_{A}\right)$, contradicting the linear independence of group characters. This shows that $f \in \mathbf{b}_{A}$.

The next result establishes a special case of Theorem 7.1.

Proposition 7.4. Suppose that $p$ is an expansive prime ideal in $R_{d}$. Then

$$
p^{-}\left(\alpha_{R_{d} / p}\right)=p^{+}\left(\alpha_{R_{d} / p}\right)=h\left(\alpha_{R_{d} / p}\right) .
$$

Proof. If $\mathfrak{p}$ is not principal, then Theorem 4.2 shows that $h\left(\alpha_{R_{d} / p}\right)=0$, and hence by (7-1) all three quantities are 0.

If $\mathfrak{p}=\langle f\rangle$ for some irreducible $f \in R_{d}$, then $f \neq 0$ since we are assuming that $\mathfrak{p}$ is expansive. For notational simplicity we shall consider only lattices of the form 
$\Lambda_{n}=n \mathbb{Z}^{d}$, but similar arguments apply for general $A$. Let $P_{n}=P_{A_{n}}$, and $\mathfrak{b}_{n}=\mathfrak{b}_{A_{n}}$. By Lemma 7.2,

$$
\left|P_{n}\left(\alpha_{R_{d} / \mathfrak{p}}\right)\right|=\left|R_{d} /\left\langle\mathfrak{p}, \mathrm{b}_{n}\right\rangle\right|=\left|R_{d} /\left\langle f, \mathrm{~b}_{n}\right\rangle\right| .
$$

Let $Q_{n}$ denote $(\mathbb{Z} / n \mathbb{Z})^{d}$. Then $R_{d} / \mathrm{b}_{n} \cong \mathbb{Z}^{Q_{i n}}$. Multiplication by $u_{j}$ on $R_{d} / \mathrm{b}_{n}$ thus corresponds to a linear map $A_{j}$ of $\mathbb{C}^{Q_{i n}}$. Let $\omega=e^{2 \pi i / n}$. As in the proof of Theorem 3.1 , the vectors $v_{\mathbf{k}} \in \mathbb{C}^{\boldsymbol{Q}_{\text {in }}}$ defined by $v_{\mathbf{k}}(\mathbf{j})=\omega \mathbf{j} \cdot \mathbf{k}$ for $\mathbf{j} \in Q_{\text {nn }}$, form an orthogonal basis for $\mathbb{C}^{Q / n}$, and are simultaneous eigenvectors for the $A_{j}$ with eigenvalues given by $A_{j}\left(v_{\mathrm{k}}\right)=\omega^{k} \cdot v_{\mathbf{k}}$. Thus the map $\varphi_{f}$ on $R_{d} / \mathbf{b}_{n}$ given by multiplication by $f$ corresponds to the linear map $f\left(A_{1}, \ldots, A_{d}\right)$ on $\mathbb{C} Q_{n n}$, whose eigenvalue for the eigenvector $v_{\mathrm{k}}$ is $f\left(\omega^{k_{1}}, \ldots, \omega^{k_{d}}\right)=f\left(\omega^{\mathrm{k}}\right)$. It follows that

$$
\begin{aligned}
\left|P_{n}\left(\alpha_{R_{d} / \mathfrak{p}}\right)\right| & =\left|R_{d} /\left\langle f, \mathrm{~b}_{n}\right\rangle\right|=\left|\left(R_{d} / b_{n}\right) / f \cdot\left(R_{d} / b_{n}\right)\right| \\
& =\left|\operatorname{det}\left(\varphi_{f}\right)\right|=\prod_{k \in Q_{j n}}\left|f\left(\omega^{\mathbf{k}}\right)\right| .
\end{aligned}
$$

Hence

$$
\frac{1}{\left|\mathbb{Z}^{d} / n \mathbb{Z}^{d}\right|} \log \left|P_{n}\left(\alpha_{R_{d} / p}\right)\right|=\frac{1}{\left|Q_{\mid n}\right|} \sum_{\mathbf{k} \in Q_{\mid n}} \log \left|f\left(e^{2 \pi \mathrm{ik} / n}\right)\right| .
$$

Since $\alpha_{R_{d} / \mathfrak{p}}$ is expansive, it follows from disjointness of $V(f)$ and $\mathbb{S}^{d}$ that $\log |f(\mathbf{s})|$ is continuous for $s \in \mathbb{S}^{d}$. The right side of (7-4) is a Riemann sum approximation to

$$
\int_{\mathbb{S}^{d}} \log |f(\mathbf{s})| d \mathbf{s}=\log M(f)=h\left(\alpha_{R_{d} / p}\right),
$$

proving that the three quantities in (7-3) are equal.

The next result shows that "commensurable" modules have the same entropy and growth rate of periodic points.

Lemma 7.5. Let $\mathfrak{p}$ be an expansive prime ideal in $R_{d}$, and $a \in R_{d} \backslash \mathfrak{p}$. If $K$ and $L$ are noetherian p-primary $R_{d}$-modules with aL $\subset K \subset L$, then $p^{ \pm}\left(\alpha_{K}\right)=p^{ \pm}\left(\alpha_{L}\right)$ and $h\left(\alpha_{K}\right)=h\left(\alpha_{L}\right)$.

Proof. Let $M$ and $N$ be p-primary $R_{d}$-modules with $a N \subset M \subset N$. Then

$$
\begin{aligned}
\left|P_{A}\left(\alpha_{N}\right)\right| & =\left|N / \mathrm{b}_{A} N\right|=\left|N /\left(M+\mathrm{b}_{A} N\right)\right| \cdot\left|\left(M+\mathrm{b}_{A} N\right) / \mathrm{b}_{A} N\right| \\
& =\left|(N / M) / \mathrm{b}_{A}(N / M)\right| \cdot\left|M /\left(M \cap \mathrm{b}_{A} N\right)\right| \\
& \leqq\left|P_{A}\left(\alpha_{N / M}\right)\right| \cdot\left|P_{A}\left(\alpha_{M}\right)\right|,
\end{aligned}
$$

the last inequality following from $\mathfrak{b}_{A} M \subset M \cap \mathfrak{b}_{A} N$. Since $\langle\mathfrak{p}, a\rangle \subset \bigcap_{\mathfrak{q} \in \operatorname{asc}(N / M)} \mathfrak{q}$, none of the prime ideals associated to $N / M$ is principal. By Theorem 6.5 , we obtain that $h\left(\alpha_{N / M}\right)=0$. It follows from (7-1) and (7-5) that $p^{ \pm}\left(\alpha_{N}\right) \leqq p^{ \pm}\left(\alpha_{M}\right)$. By the addition formula, we have that $h\left(\alpha_{N}\right)=h\left(\alpha_{N / M}\right)+h\left(\alpha_{M}\right)=h\left(\alpha_{M}\right)$.

Now assume that $M=a N$. The map $N \rightarrow M / b_{A} M$ given by multiplication by $a$ followed by the quotient map is surjective, and its kernel contains $\mathfrak{b}_{A} N$. Hence $\left|M / \mathrm{b}_{A} M\right| \leqq\left|N / \mathfrak{p}_{A} N\right|$, and (7-5) then gives

$$
\lim _{\|\Lambda\| \rightarrow \infty} \frac{1}{\left|\mathbb{Z}^{d} / \Lambda\right|}\left(\log \left|N / \mathrm{b}_{A} N\right|-\log \left|M / \mathfrak{b}_{A} M\right|\right)=0 .
$$

Since multiplication by $a$ is injective on $N$, we have that $p^{ \pm}\left(\alpha_{N}\right)=p^{ \pm}\left(\alpha_{a N}\right)$. 
Applications of this, first with $M=K$ and $N=L$, and then with $M=a L$ and $N=K$, give that $h\left(\alpha_{K}\right)=h\left(\alpha_{L}\right)$ and

$$
p^{ \pm}\left(\alpha_{L}\right) \leqq p^{ \pm}\left(\alpha_{K}\right) \leqq p^{ \pm}\left(\alpha_{a L}\right)=p^{ \pm}\left(\alpha_{L}\right) \text {. }
$$

Lemma 7.6. Let $\mathfrak{p}$ be an expansive prime ideal in $R_{d}$, and $M$ be a p-primary noetherian $R_{d}$-module. Then $p^{-}\left(\alpha_{M}\right)=p^{+}\left(\alpha_{M}\right)=h\left(\alpha_{M}\right)$.

Proof. For noetherian $R_{d}$-modules $K \subset L$, we have that $\operatorname{ann}(L) \subset \operatorname{ann}(L / K)$, and that the radical of $\operatorname{ann}(L)$ equals $\bigcap_{q \in \operatorname{asc}(L)} q=n \operatorname{asc}(L)$. It follows that $\cap \operatorname{asc}(L) \subset \cap \operatorname{asc}(L / K)$, and hence that every prime ideal associated to $L / K$ contains a prime ideal associated to $L$. From this fact we may inductively choose a prime filtration

$$
0=M_{0} \subset M_{1} \subset \cdots \subset M_{r-1} \subset M_{r}=M
$$

with $M_{j} / M_{j-1} \cong R / \mathfrak{p}_{j}$ so that $\mathfrak{p}_{j}=\mathfrak{p}$ for $1 \leqq j \leqq s$, and $\mathfrak{p}_{j} \supsetneqq \mathfrak{p}$ for $s+1 \leqq j \leqq r$, where $s \geqq 1$. For each $j$ with $s+1 \leqq j \leqq r$, choose $g_{j} \in \mathfrak{p}_{j} \backslash \mathfrak{p}$, and let $g=g_{s+1} \cdot \ldots \cdot g_{r}$. Then $g \notin p$, and $g M \subset M_{s} \subset M$. Lemma 7.5 shows that $h\left(\alpha_{M_{s}}\right)$ $=h\left(\alpha_{M}\right)$, and that $p^{ \pm}\left(\alpha_{M_{s}}\right)=p^{ \pm}\left(\alpha_{M}\right)$. This allows us to assume that $s=r$, and that $\mathfrak{p}_{j}=\mathfrak{p}$ for $1 \leqq j \leqq r$.

If $\mathfrak{p}$ is not principal, then Theorem 4.2 and the addition formula show that $h\left(\alpha_{M}\right)=0$, and we are done by $(7-1)$. So we suppose that $\mathfrak{p}=\langle f\rangle$, where $V(f) \cap \mathbb{S}^{d}=\varnothing$ by expansiveness of $\mathfrak{p}$.

We claim that $\mathfrak{b}_{A} \cap \mathfrak{p}=\mathfrak{b}_{A} \mathfrak{p}$. For suppose that $h \in \mathfrak{b}_{\Lambda} \cap \mathfrak{p}$. Then $h=f \cdot g$ for some $g \in R_{d}$ since $p=\langle f\rangle$. Now $V\left(\mathfrak{b}_{\Lambda}\right) \subset V(h)=V(f) \cup V(g)$, while $V(f) \cap \mathbb{S}^{d}=\varnothing$ and $V\left(\mathfrak{b}_{A}\right) \subset \mathbb{S}^{d}$. Thus $V\left(\mathfrak{b}_{A}\right) \subset V(g)$, so $g$ vanishes on $V\left(\mathfrak{b}_{A}\right)$, and hence $g \in \mathfrak{b}_{A}$ by Lemma 7.3. Thus $h=f \cdot g \in \mathfrak{b}_{A} \mathfrak{p}$, as claimed.

We next show that $M_{j} \cap \mathrm{b}_{A} M=\mathrm{b}_{A} M_{j}$ for $1 \leqq j \leqq r$. If not, there is an $x \in\left(M_{j} \cap \mathfrak{b}_{A} M\right) \backslash \mathfrak{b}_{A} M_{j}$. Choose $k>j$ minimal so that $x \in \mathfrak{b}_{A} M_{k}$. Then there are $x_{1}, \ldots, x_{n} \in M_{k}$ and $f_{1}, \ldots, f_{n} \in \mathrm{b}_{\Lambda}$ with $x=\sum_{i=1}^{n} f_{i} x_{i}$, and $\left\{x_{1}, \ldots, x_{n}\right\} \not M_{k-1}$ by minimality of $k$. Choose $z \in M_{k}$ so that the map $f \mapsto f z+M_{k-1}$ induces an isomorphism of $R_{d} / \mathfrak{p}$ with $M_{k} / M_{k-1}$. Then there are $g_{i} \in R_{d}$ with $x_{i}-g_{i} z=y_{i} \in M_{k-1}$ for $1 \leqq i \leqq n$. Since $\left\{x_{1}, \ldots, x_{n}\right\} \not M_{k-1}$, it follows that $\left(g_{1}, \ldots, g_{n}\right\} \not \subset \mathfrak{p}$, while

$$
\sum_{i=1}^{n} f_{i} g_{i} z=\sum_{i=1}^{n} f_{i}\left(x_{i}-y_{i}\right) \in M_{k-1}
$$

since $x=\sum_{i=1}^{n} f_{i} x_{i} \in M_{j} \subset M_{k-1}$. Hence

$$
\sum_{i=1}^{n} f_{i} g_{i} \in \mathfrak{b}_{A} \cap \mathfrak{p}=\mathbf{b}_{\Lambda} \mathfrak{p}
$$

so there are $h_{1}, \ldots, h_{m} \in \mathfrak{b}_{\Lambda}$ and $k_{1}, \ldots, k_{m} \in \mathfrak{p}$ such that $\sum_{i=1}^{n} f_{i} g_{i}=\sum_{l=1}^{m} h_{l} k_{l}$. Thus

$$
x=\sum_{l=1}^{n} h_{l}\left(k_{l} z\right)+\sum_{i=1}^{n} f_{i} y_{i} \in \mathfrak{b}_{A} M_{k-1}
$$

contradicting minimality of $k$. We conclude that $M_{j} \cap \mathfrak{b}_{A} M=\mathfrak{b}_{A} M_{j}$ for $1 \leqq j \leqq r$. 
It now follows from (7-5) that

$$
\begin{aligned}
\left|P_{A}\left(\alpha_{M}\right)\right| & =\left|M / \mathrm{b}_{A} M\right|=\left|\left(M_{r} / M_{r-1}\right) / \mathrm{b}_{\Lambda}\left(M_{r} / M_{r-1}\right)\right| \cdot\left|M_{r-1} / \mathrm{b}_{A} M_{r-1}\right| \\
& =\cdots=\prod_{j=1}^{r}\left|P_{A}\left(\alpha_{M_{j} / M_{3-1}}\right)\right|=\left|P_{A}\left(\alpha_{R_{d} / p}\right)\right|^{r} .
\end{aligned}
$$

Since $h\left(\alpha_{M}\right)=\sum_{j=1}^{r} h\left(\alpha_{M_{J} / M_{j-1}}\right)=r h\left(\alpha_{R_{d} / p}\right)$, the result now follows from Proposition 7.4 .

Proof of Theorem 7.1. Since $\alpha_{M}$ is expansive, $M$ is noetherian. Let $\operatorname{asc}(M)=$ $\left\{\mathbf{p}_{1}, \ldots, \mathbf{p}_{n}\right\}$, so that each $\mathbf{p}_{j}$ is expansive [Sc2, Thm. 3.9]. By the primary decomposition theorem, there are submodules $M_{1}, \ldots, M_{n}$ of $M$ such that $M / M_{j}$ is $\mathfrak{p}_{j}$-primary, and $\bigcap_{j=1}^{n} M_{j}=\{0\}$. It follows that the diagonal map

$$
\theta: M \rightarrow \bigoplus_{j=1}^{n}\left(M / M_{j}\right)=L
$$

is injective. The dual map $X_{L}=\prod_{j=1}^{n} X_{M / M_{j}} \rightarrow X_{M}$ is therefore surjective. Denote its kernel by $Y$. The addition formula shows that

$$
\sum_{j=1}^{n} h\left(\alpha_{M / M_{j}}\right)=h\left(\alpha_{L}\right)=h\left(\alpha_{M}\right)+h\left(\alpha_{Y}\right)
$$

where $\alpha_{Y}$ denotes the restriction of $\alpha$ to $Y$.

We claim that $h\left(\alpha_{Y}\right)=0$. We first observe that if an $R_{d}$-module $N$ is the sum (not necessarily direct) of a finite collection $\left\{N_{k}\right\}$ of submodules, and if $h\left(\alpha_{N_{k}}\right)=0$ for each $k$, then $h\left(\alpha_{N}\right)=0$. For the addition map $\oplus_{k} N_{k} \rightarrow \sum_{k} N_{k}=N$ is surjective, so that $\alpha_{N}$ is a subsystem of $\prod_{k} \alpha_{N_{k}}$. Since $h\left(\prod_{k} \alpha_{N_{k}}\right)=\sum_{k} h\left(\alpha_{N_{k}}\right)=0$, we obtain that $h\left(\alpha_{N}\right)=0$, as required. Now the dual of $Y$ is $L / \theta(M)=\left[\oplus\left(M / M_{j}\right)\right] / \theta(M)$, so that our observation allows us to confine our attention to a single summand. If $\mathfrak{p}_{j}$ is not principal, then any prime annihilator of an element in $\left(0, \ldots, a+M_{j}, \ldots, 0\right)$ $+\theta(M)$ contains $\mathfrak{p}_{j}$, hence is not principal. If $\mathfrak{p}_{j}$ is principal, then such an annihilator contains $\mathfrak{p}_{j}+\prod_{k \neq j} \mathfrak{p}_{k}$, and is again not principal since $\mathfrak{p}_{j}$ is a minimal non-zero prime and the $\mathfrak{p}_{k}$ are distinct and non-zero. Thus the primes associated to each summand $\left(0, \ldots, a+M_{j}, \ldots, 0\right)+\theta(M)$ are all non-principal, and Theorem 4.2 combined with our observation show that $h\left(\alpha_{Y}\right)=0$. It follows from (7-1) that $p^{-}\left(\alpha_{Y}\right)=p^{+}\left(\alpha_{Y}\right)=h\left(\alpha_{Y}\right)=0$, and hence from (7-5) that $p^{ \pm}\left(\alpha_{L}\right)=p^{ \pm}\left(\alpha_{M}\right)$ and that $h\left(\alpha_{L}\right)=h\left(\alpha_{M}\right)$.

Now Lemma 7.6 shows that $p^{-}\left(\alpha_{M / M_{j}}\right)=p^{+}\left(\alpha_{M / M}\right)=h\left(\alpha_{M / M}\right)$. Since the limit

$$
\lim _{\|\boldsymbol{\Lambda}\| \rightarrow \infty} \frac{1}{\left|\mathbb{Z}^{d} / \Lambda\right|} \log \left|P_{\Lambda}\left(\alpha_{M / M}\right)\right|
$$

exists for each $j$, both $p^{-}$and $p^{+}$add over the direct products, so that

$$
\begin{aligned}
p^{-}\left(\alpha_{L}\right) & =p^{-}\left(\alpha_{\oplus\left(M / M_{j}\right)}\right)=\sum_{j} p^{-}\left(\alpha_{M / M_{j}}\right) \\
& =\sum_{j} p^{+}\left(\alpha_{M / M_{j}}\right)+p^{+}\left(\alpha_{\oplus\left(M / M_{j}\right)}\right)=p^{+}\left(\alpha_{L}\right) .
\end{aligned}
$$


Combining these, and noting that $\alpha_{L}$ is expansive since each $\alpha_{M / M_{2}}$, is, we obtain that

$$
\begin{aligned}
h\left(\alpha_{M}\right) & =h\left(\alpha_{L}\right)=\sum_{j} h\left(\alpha_{M / M}\right)=p^{-}\left(\alpha_{L}\right) \\
& =p^{+}\left(\alpha_{L}\right) \leqq h\left(\alpha_{L}\right)=h\left(\alpha_{M}\right),
\end{aligned}
$$

concluding the proof.

\section{Appendix A. Entropies for actions}

Let $\alpha$ be a $\mathbb{Z}^{d}$-action on the compact metrizable group $X$. It is useful to have a variety of ways to compute the entropy of $\alpha$. Here we show that five common methods coincide, and also prove a technical replacement for subadditivity needed in the proof of Theorem 3.1. We use the notation and development in [W], merely sketching the changes required for our situation.

Recall from $\$ 2$ that if $Q=\prod_{j=1}^{d}\left\{b_{j}, \ldots, b_{j}+l_{j}-1\right\}$ is a rectangle in $\mathbb{Z}^{d}$, the its girth $g(Q)$ is

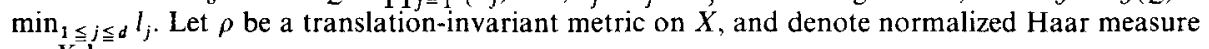
on $X$ by $\mu$.

If $\mathscr{U}$ is an open cover of $X$, let $N(\mathscr{U})$ be the number of elements in the smallest subcover of $\mathscr{U}$. Then $\log N(\cdot)$ is subadditive in the sense that $\log N(\mathscr{U} \vee \mathscr{V}) \leqq \log N(\mathscr{U})+\log N(\mathscr{V})$. Put

$$
h_{\text {cover }}(\alpha)=\sup _{\mathscr{U}} \lim _{g(Q) \rightarrow \infty} \frac{1}{|Q|} \log N\left(\bigvee_{\mathbf{j} \in Q} \alpha^{-\mathrm{j} \mathscr{U}}\right) \text {, }
$$

where the limit exists by subadditivity of $\log N(\cdot)$ using a standard Folner argument.

A set $E \subset X$ is called $(Q, \varepsilon)$-spanning for $\alpha$ if for every $x \in X$ there is a $y \in E$ such that $\rho\left(\alpha^{\mathrm{j}} x, \alpha^{\mathrm{j}} y\right)<\varepsilon$ for all $\mathbf{j} \in Q$. Let $r_{Q}(\varepsilon, \alpha)$ be the smallest cardinality of a $(Q, \varepsilon)$-spanning set, and put

$$
r(\varepsilon, \alpha)=\limsup _{g(Q) \rightarrow \infty} \frac{1}{|Q|} \log r_{Q}(\varepsilon, \alpha), \quad h_{\text {span }}(\alpha)=\lim _{\varepsilon \rightarrow 0} r(\varepsilon, \alpha) .
$$

Dually, $F \subset X$ is $(Q, \varepsilon)$-separated for $\alpha$ if for distinct $x, y \in F$ there is a $\mathbf{j} \in Q$ such that $\rho\left(x^{j} x, \alpha^{j} y\right) \geqq \varepsilon$. Let $s_{Q}(\varepsilon, \alpha)$ be the largest cardinality of a $(Q, \varepsilon)$-separated set, and put

$$
s(\varepsilon, \alpha)=\limsup _{g(Q) \rightarrow \infty} \frac{1}{|Q|} \log s_{Q}(\varepsilon, \alpha), \quad h_{\mathrm{sep}}(\alpha)=\lim _{\varepsilon \rightarrow 0} s(\varepsilon, \alpha) .
$$

Let $B(\varepsilon)$ be the $\varepsilon$-ball around the identity of $X$, and put $D_{Q}(\varepsilon, \alpha)=\bigcap_{\mathbf{j} \in Q^{\alpha^{-j}} B(\varepsilon) \text {. Define }}$

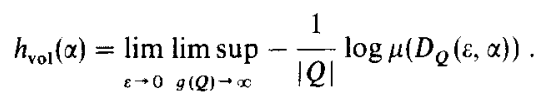

Finally, let $h_{\mu}(\alpha)$ be the measure-theoretic entropy of $\alpha$ with respect to Haar measure, as developed in [C].

Theorem A.1. Let $\alpha$ be a $\mathbb{Z}^{d}$ action by automorphisms of a compact metrizable group. Then the five entropies above coincide. We denote their common value by $h(\alpha)$.

Proof. Let $\mathscr{U}_{\varepsilon}$ be the open cover of all $\varepsilon$-balls in $X$. The argument of [W, Corollary 7.7.1] shows

$$
N\left(\bigvee_{\mathbf{j} \in Q} \alpha^{-\mathbf{j} \mathscr{U}_{2 \varepsilon}}\right) \leqq r_{Q}(\varepsilon, \alpha) \leqq s_{Q}(\varepsilon, \alpha) \leqq N\left(\bigvee_{\mathbf{j} \in Q} \alpha^{-\mathbf{j} \mathscr{U}_{\varepsilon / 2}}\right)
$$

Since $\operatorname{diam}\left(\mathscr{U}_{\varepsilon}\right)=2 \varepsilon \rightarrow 0$ as $\varepsilon \rightarrow 0$, it follows that $h_{\text {cover }}(\alpha)=h_{\text {span }}(\alpha)=h_{\text {sep }}(\alpha)$. That $h_{\mu}(\alpha) \leqq h_{\text {sover }}(\alpha)$ follows from the variational principle for $\mathbb{Z}^{d}$-actions [E] [Ms]. If $\xi$ is a finite measurable partition of $X$ into sets of diameter $<\varepsilon$, then as in [W, Thm. 8.11],

$$
-\log \mu\left(D_{Q}(\varepsilon, \alpha)\right) \leqq H_{\mu}\left(\bigvee_{\mathbf{j} \in Q} \alpha^{-\mathbf{j} \xi}\right)
$$


hence $h_{\mathrm{vol}}(\alpha) \leqq h_{\mu}(\alpha)$. If $E$ is a $(Q, \varepsilon)$-separated set of cardinality $s_{Q}(\varepsilon, \alpha)$, then the sets $\left\{x D_{Q}(\varepsilon / 2, \alpha)\right.$ : $x \in E\}$ are disjoint, so

$$
s_{Q}(\varepsilon, \alpha) \leqq 1 / \mu\left(D_{Q}(\varepsilon, \alpha)\right)
$$

It follows that $h_{\text {sep }}(\alpha) \leqq h_{\mathrm{vol}}(\alpha)$, completing the proof.

The reason for using lim sup's instead of limits to define $h_{\text {span }}(\alpha)$ and $h_{\text {sep }}(\alpha)$ is that $\log r_{Q}(\varepsilon, \alpha)$ and $\log \mathrm{s}_{Q}(\varepsilon, \alpha)$ need not be subadditive over disjoint rectangles. The following result, needed in the proof for the upper bound part of Theorem 3.1, is a partial replacement for subadditivity.

Proposition A.2. Suppose that for some rectangle $Q_{0}$ we have

$$
\frac{1}{\left|Q_{0}\right|} \log s_{Q_{0}}(\varepsilon, \alpha) \leqq B
$$

Then $r(4 \varepsilon, \alpha) \leqq s(4 \varepsilon, \alpha) \leqq B$.

Proof. For a large rectangle $Q$, there is a $\Lambda \subset \mathbb{Z}^{d}$ such that the sets $\left\{Q_{0}+\mathbf{k}: \mathbf{k} \in \boldsymbol{A}\right\}$ are disjoint, their union $Q_{1} \supset Q$, and $\left|Q_{1}\right| /|Q| \rightarrow 1$ as $g(Q) \rightarrow \infty$. Thus $|Q| /|A| \rightarrow\left|Q_{0}\right|$ as $g(Q) \rightarrow \infty$. By (A-3),

$$
\begin{aligned}
& s_{Q}(4 \varepsilon, \alpha) \leqq s_{Q_{1}}(4 \varepsilon, \alpha) \leqq N\left(\bigvee_{\mathbf{j} \in Q_{1}} \alpha^{-\mathbf{j} \mathscr{U}_{2 \varepsilon}}\right) \leqq \prod_{\mathbf{k} \in A} N\left(\underset{\mathbf{j} \in Q_{0}+\mathbf{k}}{\bigvee} \alpha^{-\mathbf{j} \mathscr{U}_{2 \varepsilon}}\right) \\
& =\left[N\left(\bigvee_{\mathbf{j} \in Q_{0}} \alpha^{-\mathbf{j}} \mathscr{U}_{2 \varepsilon}\right)\right]^{|\Lambda|} \leqq r_{Q_{0}}(\varepsilon, \alpha)^{|\Lambda|} \leqq s_{Q_{0}}(\varepsilon, \alpha)^{|A|} \text {. }
\end{aligned}
$$

Hence

$$
\frac{1}{|Q|} \log s_{Q}(4 \varepsilon, \alpha) \leqq \frac{|A|}{|Q|} \log s_{Q_{0}}(\varepsilon, \alpha) \rightarrow \frac{1}{\left|Q_{0}\right|} \log s_{Q_{0}}(\varepsilon, \alpha) \leqq B
$$

as $g(Q) \rightarrow \infty$, which yields the result.

\section{Appendix B. The addition formula}

If $\alpha$ is a $\mathbb{Z}^{d}$-action on the compact group $X$, and $Y$ is an $\alpha$-invariant normal subgroup of $X$, let $\alpha_{Y}$ denote the restriction of $\alpha$ to $Y$, and $\alpha_{X / Y}$ be the induced action on $X / Y$. The addition formula for subgroups states

$$
h(\alpha)=h\left(\alpha_{X / Y}\right)+h\left(\alpha_{Y}\right) .
$$

Since $\alpha$ can be regarded as a skew product with base action $\alpha_{X / Y}$ and an affine fiber action whose automorphism part is $\alpha_{Y}$, (B-1) will follow from a more general addition formula for skew products with affine fiber actions. When $d=1$, the addition formula for subgroups was proven by Yuzvinskii [Yz1]. His arguments were adapted by Thomas [T1] for skew products. These papers contain the ideas needed to handle the case $d>1$ as well, and essentially everything in this section is due to those authors.

In order to describe the addition formula for skew products, suppose that $\beta$ is a measurepreserving action of $\mathbb{Z}^{d}$ on a Lebesgue space $(\Omega, \mathscr{M}, v)$, and that $\alpha$ is a $\mathbb{Z}^{d}$-action by automorphisms of a compact metrizable group $Y$ equipped with the Borel $\sigma$-algebra and Haar measure $\mu$. We use multiplicative notation for $Y$. A cocycle $\sigma$ for $\beta$ and $\alpha$ is a measurable map $\sigma: \mathbb{Z}^{d} \times \Omega \rightarrow Y$ such that

$$
\sigma(\mathbf{m}+\mathbf{n}, \omega)=\alpha^{\mathbf{m}}(\sigma(\mathbf{n}, \omega)) \cdot \sigma\left(\mathbf{m}, \beta^{\mathbf{n}} \omega\right)
$$

for all $\mathbf{m}, \mathbf{n} \in \mathbb{Z}^{d}$ and $\omega \in \Omega$. This cocycle equation provides the consistency to define the skew product action $\beta \times{ }_{\sigma} \alpha$ on $(\Omega \times Y, \nu \times \mu)$ by

$$
\left(\beta \times{ }_{\sigma} \alpha\right)^{\mathbf{n}}(\omega, y)=\left(\beta^{\mathbf{n}} \omega,\left(\alpha^{\mathbf{n}} y\right) \cdot \sigma(\mathbf{n}, \omega)\right) .
$$

When $\sigma \equiv 1_{Y}$, we obtain the direct product action $\beta \times \alpha$. 
Theorem B.1. (The addition formula). If $\beta$ is a measure-preserving $\mathbb{Z}^{d}$-action on $(\Omega, v), \alpha$ is an algebraic $\mathbb{Z}^{d}$-action on a compact group $Y$ with Haar measure $\mu$, and $\sigma: \mathbb{Z}^{d} \times \Omega \rightarrow Y$ is a cocycle for $\beta$ and $\alpha$, then

$$
h_{v \times \mu}\left(\beta \times{ }_{\sigma} \alpha\right)=h_{v}(\beta)+h_{\mu}(\alpha) .
$$

Corollary B.2. If $\alpha$ is a $\mathbb{Z}^{d}$-action on a compact group $X$, and $Y$ is a closed, normal, $\alpha$-invariant subgroup of $X$, then

$$
h(\alpha)=h\left(\alpha_{X / Y}\right)+h\left(\alpha_{Y}\right) .
$$

Proof of the Corollary. Use a Borel cross-section to the natural quotient map $X \rightarrow X / Y$ to construct a cocycle $\sigma: \mathbb{Z}^{d} \times(X / Y) \rightarrow Y$ such that $\alpha$ is measurable isomorphic to $\alpha_{X / Y} \times_{\sigma} \alpha_{Y}$. Apply the addition formula.

Our proof of (B-3) recapitulates that of Yuzvinskii for $d=1$, augmented by some improvements due to Thomas. We use the language and results of Rohlin's theory of measurable partitions. An excellent account of this is contained in Parry's book [P], which the reader should consult for any unexplained notation or terminology.

If $\xi$ is a mesurable partition of $Y$ and $y \in Y$, let $\xi \cdot y$ denote the right translate of the elements of $\xi$ by $y$. Let $\left\{\xi_{m}\right\}$ be a sequence of finite measurable partitions of $Y$ increasing to the partition of $Y$ into points. For a rectangle $Q$ in $\mathbb{Z}^{d}$ and $\omega \in \Omega$, define

$$
\xi_{m}^{Q}=\bigvee_{\mathbf{j} \in Q} \alpha^{-\mathbf{j}} \xi_{m}, \quad \xi_{m, \omega}^{Q}=\bigvee_{\mathbf{j} \in Q}\left(\alpha-\mathbf{j} \xi_{m}\right) \cdot \sigma(-\mathbf{j}, \omega)
$$

Standard entropy calculations show that

$$
\begin{aligned}
& h_{v \times \mu}(\beta \times \alpha)=h_{v}(\beta)+\lim _{m \rightarrow \infty} \limsup _{g(Q) \rightarrow \infty} \frac{1}{|Q|} \int_{\Omega} H_{\mu}\left(\xi_{m}^{Q}\right) d v(\omega), \\
& h_{v \times \mu}\left(\beta \times \times_{\sigma} \alpha\right)=h_{v}(\beta)+\lim _{m \rightarrow \infty} \limsup _{g(Q) \rightarrow \infty} \frac{1}{|Q|} \int_{\Omega} H_{\mu}\left(\xi_{m, \omega}^{Q}\right) d v(\omega) .
\end{aligned}
$$

The addition formula holds trivially if $h_{v}(\beta)=\infty$, so we will assume from now on that $h_{v}(\beta)<\infty$. It therefore suffices to show that the fiber contributions on the right sides are equal, i.e. that translations on the fibers driven by the cocycle do not affect entropy. Yuzvinskii's idea is to show that there are certain basic groups for which "rigid" partitions exist so that this holds, and then build up a general compact group from basic groups using processes that preserve the addition formula.

Definition B.3 (Yuzvinskii). A compact group $Y$ with Haar measure $\mu$ is rigid if there is a sequence $\left\{\xi_{m}\right\}$ of finite measurable partitions of $Y$ and a constant $K$ so that

$$
H_{\mu}\left(\xi_{m} \mid \xi_{m} \cdot y\right)+H\left(\xi_{m} \cdot y \mid \xi_{m}\right) \leqq K
$$

for every $m \geqq 1$ and $y \in Y$.

Say that "the addition formula holds for $\alpha$ " if for every base action $\beta$ and every cocycle $\sigma$ we have (B-3). Say "the addition formula holds for $Y$ " if it holds for every $\mathbb{Z}^{d}$ action on $Y$. The importance of rigidity is the following key result.

Lemma B.4. The addition formula holds for all rigid groups.

Proof. Let $Y$ be rigid, and $\left\{\xi_{m}\right\}, K$ be as in Def B.3. The same calculation as in [Yz1, Thm. 7.4] shows that

$$
\begin{aligned}
\left|H_{\mu}\left(\xi_{m}^{Q}\right)-H_{\mu}\left(\xi_{m, \omega}^{Q}\right)\right| & \leqq \sum_{\mathbf{j} \in Q}\left[H_{\mu}\left(\xi_{m} \mid \xi_{m} \cdot \alpha^{\mathbf{j}} \sigma(-\mathbf{j}, \omega)\right)+H_{\mu}\left(\xi_{m} \cdot \alpha^{\mathbf{j}} \sigma(-\mathbf{j}, \omega) \mid \xi_{m}\right)\right] \\
& \leqq 2 K|Q|,
\end{aligned}
$$

where $K$ does not depend on $\alpha$. Let $\alpha^{k}$ denote the $\mathbb{Z}^{d}$-action given by $\left(\alpha^{k}\right)^{\mathbf{n}}=\alpha^{k \mathrm{n}}$. Replace in the above $\alpha$ by $\alpha^{k}, \beta$ by $\beta^{k}$, and $\sigma$ by a suitable cocycle $\sigma_{k}$ so that $\left(\beta \times_{\sigma} \alpha\right)^{k}=\beta^{k} \times_{\sigma_{k}} \alpha^{k}$. Then dividing 
(B-4) by $|Q|$, taking limsup $\operatorname{sig}_{g(Q) \rightarrow \infty}$ and then $\lim _{m \rightarrow 0}$, we obtain that

$$
\left.k^{d} \mid h_{v \times \mu}(\beta \times \alpha)-h_{v \times \mu}\left(\beta \times{ }_{\sigma} \alpha\right)\right\} \leqq 2 K .
$$

Since $k$ is arbitrary, the result follows.

The next lemma gives a rich supply of rigid groups.

Lemma B.5. Totally disconnected compact groups, compact Lie groups, and solenoids are rigid.

Proof. Rigidity for totally disconnected compact groups and solenoids is shown in Theorems 7.2 and 7.3 of $[\mathrm{Yz} 1]$. There is a minor error in the proof for the solenoid case, reproduced in [T1, Thm. 2.6]. The increasing sequence $\left\{\eta_{n}\right\}$ of partitions constructed need not converge to the partition into points. For example, using the notations from the proof of Thm. 7.3 in [Yz1], let $A$ $=\mathbb{Z}[1 / 2]^{\prime}, l=1, F_{n}=\left(2^{-n} \mathbb{Z}\right)^{n}, p_{n}: A \rightarrow A / F_{n} \cong \mathbb{T}, k=2^{n-1}$ at stage $n, m(n)=2^{n-1}$, and $\eta_{n}$ $=p_{n}^{-1}\left(\zeta_{m(n)}\right)$, where $\zeta_{m}$ divides $T$ into $m$ equal intervals. For $n \geqq 1$ and every $C \in \eta_{n}$, we have $p_{1}(C)$ $=A / F_{1}$, so $\bigvee_{n=1}^{\infty} \eta_{n} \neq \varepsilon$, contrary to the claim. The proof can be easily fixed by choosing $k$ twice as large as needed at each stage, which forces $\operatorname{diam}\left(\eta_{n}\right) \rightarrow 0$.

Thomas [T1, Thm. 2.5] proved that compact Lie groups are rigid.

The next three lemmas provide machinery to build up general compact groups from rigid ones. They can be summarized by saying that the addition formula is preserved under extensions, inverse limits, and direct products.

Lemma B.6. Suppose $\alpha$ is a $\mathbb{Z}^{d}$-action on $X$, and that $Y$ is a closed, normal, $\alpha$-invariant subgroup of $X$. If the addition formula holds for $\alpha_{X / Y}$ and for $\alpha_{Y}$, then it holds for $\alpha$.

Proof. Same as for $d=1$ in $[\mathrm{T} 1, \S 1.4]$.

Lemma B.7. Suppose $\alpha$ is a $\mathbb{Z}^{d}$-action on $X$, and that $X=Y_{0} \supset Y_{1} \supset Y_{2} \supset \ldots$ are $\alpha$-invariant closed subgroups with $Y_{i+1}$ normal in $Y_{i}$ for $i \geqq 0$, and such that $\bigcap_{n=0}^{\infty} Y_{n}=\{1\}$. If the addition formula holds for each $\alpha_{X / Y_{n}}$, then it holds for $\alpha$.

Proof. Same as for $d=1$ in $[\mathrm{T} 1, \S 1.5]$.

Lemma B.8. Suppose I is a finite or countable set, and that for every $i \in I$ there is a $\mathbb{Z}^{d}$-action $\alpha_{i}$ on $X_{i}$ for which the addition formula holds. Then it also holds for the direct product action $\prod_{i \in I} \alpha_{i}$ on $\prod_{i \in I} X_{i}$.

Proof. For $I$ finite this follows by induction from Lemma B.6. For $I=\{1,2, \ldots\}$ apply Lemma B.7 with $Y_{n}=\prod_{i=n+1}^{\infty} X_{i}$ and the finite product case.

Proof of the addition formula. We will build up a general $\mathbb{Z}^{d}$-action from those obeying the addition formula using extensions, inverse limits, and skew products, processes which preserve the formula.

Suppose that $\alpha$ is a $\mathbb{Z}^{d}$-action on the compact group $Y$. Let $Y^{0}$ denote the connected component of the identity. Then $Y^{0}$ is closed, normal, and $\alpha$-invariant. Since $Y / Y^{0}$ is totally disconnected, hence rigid by Lemma B.5, the Lemmas B.4 and B.6 allow us to restrict ourselves to the case where $Y$ is connected.

Next suppose that $Y$ is connected and centerless. By $[Y z 1, \S 4.1], Y$ is a direct product $\prod_{i \in I} Y_{i}$, where each $Y_{i}$ is an algebraically simple $L$ ie group. Furthermore, if $\mathbf{n} \in \mathbb{Z}^{d}$ and $i \in I$, then $\alpha^{\mathbf{n}}\left(Y_{i}\right)=Y_{j}$ for some $j \in I$. Thus $\mathbb{Z}^{d}$ acts on $I$. Decomposing $I$ into corresponding equivalence classes and using Lemma B.8, we can assume this action is transitive on $I$. Fix $i_{0} \in I$, and put $A=\left\{\mathbf{n} \in \mathbb{Z}^{d}: \alpha^{\mathrm{n}}\left(Y_{i_{0}}\right)=Y_{i_{0}}\right\}$. If $A=\{\mathbf{0}\}$, then $\alpha$ is isomorphic to a group shift on $Y_{i_{0}}$, and a measurable isomorphism of $\beta \times{ }_{\sigma} \alpha$ with $\beta \times \alpha$ can be constructed exactly as in [T1, Thm. 3.2]. If $A \neq\{0\}$. then by a change of basis we can assume that $A$ is generated by $r_{1} \mathbf{e}_{1}, \ldots, r_{c} \mathbf{e}_{c}$ for some $1 \leqq c \leqq d$.

Let $\mathbf{0}_{c} \in \mathbb{Z}^{c}$ and $\mathbf{0}_{d-c} \in \mathbb{Z}^{d-c}$ be the zero elements, and put

$$
\bar{Y}=\prod\left\{\alpha^{\mathbf{n}}\left(Y_{i_{0}}\right): \mathbf{0} \leqq \mathbf{n} \leqq r_{i} \mathbf{e}_{1}+\cdots+r_{c} \mathbf{e}_{\mathbf{c}}\right\} .
$$

Then $\bar{Y}$ is invariant under $\alpha^{\mathbf{j}}$ for every $\mathbf{j} \in \mathbb{Z}^{c} \times\left\{\mathbf{0}_{d-c}\right\}$, and the subgroups $\left\{\alpha^{\mathbf{k}}(\bar{Y}): \mathbf{k} \in\left\{\mathbf{0}_{c}\right\} \times \mathbb{Z}^{d-c}\right\}$ are independent. By Lemma B.5, $\bar{Y}$ is rigid, so there are $\left\{\xi_{m}\right\}$ and $K$ as in Definition B.3. Let $Q=Q_{c} \times Q_{d-c}$ be a product of rectangles and use the same notation as in the proof of Lemma B.4. 
Independence and a rigidity argument imply that

$$
\begin{aligned}
\left|H_{\mu}\left(\xi_{m}^{Q}\right)-H_{\mu}\left(\xi_{m, \omega}^{Q}\right)\right| & \leqq \sum_{\mathbf{k} \in Q_{d-c}}\left|H_{\mu}\left(\xi_{m}^{Q_{c}+\mathbf{k}}\right)-H_{\mu}\left(\xi_{m, \omega}^{Q_{c}+\mathbf{k}}\right)\right| \\
& \leq\left|Q_{d-c}\right| \cdot 2 K\left|Q_{c}\right|=2 K|Q|
\end{aligned}
$$

The proof for the centerless case is now completed as in Lemma B.4.

If $Y$ is connected, and $Z$ is its center, then $Y / Z$ is connected and centerless [YZ1, §1. 1.3]. By the above, we have reduced the problem to the case where $Y$ is abelian.

Let $M=\hat{Y}$ be the dual group, which as in $\S 2$ is an $R_{d}$-module. If $M_{0}$ is the torsion submodule of $M$, then $M / M_{0}$ is torsion-free, and it suffices by Lemma B.6 to consider the torsion and torsionfree cases separately. If $M$ is torsion-free, then every finitely generated submodule $F$ is free, so the addition formula holds for $Y / F^{\perp}$ by an explicit isomorphism [T1, Thm. 3.2]. Since $M$ is a countable increasing union of finitely generated submodules, the torsion-free case is completed by an appeal to Lemma B.7. Finally, suppose that $M$ is a torsion module, and let $F$ be a finitely generated submodule. Then $Y / F^{\perp}$ is a solenoid, so the addition formula holds here. Since $M$ is the countable increasing union of finitely generated submodules, another appeal to Lemma B.7 completes the proof.

\section{References}

[A] Ahlfors, L.V.: Complex Analysis, 2nd edn. New York: McGraw-Hill 1966

[AM] Atiyah, M., Macdonald, I.: Introduction to Commutative Algebra. Reading: AddisonWesley 1969

[Bg] Berg, K.R.: Convolutions of invariant measures, maximal entropy. Math. Syst. Theory 3, 146-150 (1969)

[Bw] Bowen, R.: Entropy for group endomorphisms and homogeneous spaces. Trans. Am. Math. Soc. 153, 401-414 (1971)

[By1] Boyd, D.: Kronecker's theorem and Lehmer's problem for polynomials in several variables. J. Number Theory 13, 116-121 (1981)

[By2] Boyd, D.: Speculations concerning the range of Mahler's measure. Can. Math. Bull. 24, 453-469 (1981)

[C] Conze, J.P.: Entropie d'un groupe abélian de transformations. Z. Wahrscheinlichkeitsth. Verw. Geb. 25, 11-30 (1972)

[D] Dobrowolski, E., Lawton, W., Schinzel, A.: On a problem of Lehmer. (Studies in Pure Math., pp. 133-144). Basel: Birkhäuser 1983

[E] Elsanousi, S.A.: A variational principle for the pressure of a continuous $\mathbb{Z}^{2}$-action on a compact metric space. Am. J. Math. 99, 77-106 (1977)

[Km] Kaminski, B.: Mixing properties of two-dimensional dynamical systems with completely positive entropy. Bull. Pol. Acad. Sci., Math. 27, 453-463 (1980)

[Kt] Kato, T.: Perturbation Theory for Linear Operators. New York: Springer 1966

[KS1] Kitchens, B., Schmidt, K.: Automorphisms of compact groups. Ergodic Theory Dyn. Syst. 9, 691-735 (1989)

[KS2] Kitchens, B., Schmidt, K.: Periodic points, decidability and Markov subgroups. (Lecture Notes in Math., Vol. 1342 pp. 440-454). Berlin-Heidelberg-New York: Springer 1988

[Lg] Lang, S.: Algebra (2nd Ed.). Reading: Addison-Wesley 1984

[Lw] Lawton, W.M.: A problem of Boyd concerning geometric means of polynomials. J. Number Theory 16, 356-362 (1983)

[Ld] Ledrappier, F.: Un champ markovian peut être d'entropie nulle et mélangeant. C. $R$. Acad. Sc. Paris. Ser. A 2807, 561-562 (1978)

[Lh] Lehmer, D.H.: Factorization of cyclotomic polynomials. Ann. Math. 34, 461-479 (1933).

[Lnl] Lind, D.A.: Translation invariant sigma algebras on groups. Proc. Am. Math. Soc. 42, 218-221 (1974) 
[Ln2] Lind, D.A.: Ergodic automorphisms of the infinite torus are Bernoulli. Isr. J. Math. 17, $162-168(1974)$

[Ln3] Lind, D.A.: The structure of skew products with ergodic group automorphisms. Isr. J. Math. 28, 205-248 (1977)

[Ln4] Lind, D.A.: Dynamical properties of quasihyperbolic toral automorphisms. Ergodic Theory Dyn. Syst. 2, 48-68 (1982)

[LW] Lind, D., Ward, T.: Automorphisms of solenoids and p-adic entropy. Ergodic Theory Dyn. Syst. 8, 411-419 (1988)

[Mh1] Mahler, K.: An application of Jensen's formula to polynomials. Mathematika 7, $98-100(1960)$

[Mh2] Mahler, K.: On some inequalities for polynomials in several variables. J. London Math. Soc. 37, 341-344 (1962)

[Mt] Matsumura, H.: Commutative Algebra. New York: Benjamin 1970

[Ms] Misiurewicz, M:: A short proof of the variational principle for a $\mathbb{Z}_{+}^{N}$-action on a compact space. Asterisque 40, 147-157 (1975)

[P] Parry, W.: Entropy and Generators in Ergodic Theory. New York: Benjamin 1969

[Rh] Rohlin, V.A.: Metric properties of endomorphisms of compact commutative groups. Am. Math. Soc. Transl., Ser. 2 64, 244-252 (1967)

[Rd] Rudin, W.: Real and Complex Analysis. New York: McGraw-Hill 1966

[Scl] Schmidt, K.: Mixing automorphisms of compact groups and a theorem by Kurt Mahler. Pac. J. Math. 137, 371-385 (1989)

[Sc2] Schmidt, K.: Automorphisms of compact abelian groups and affine varieties. Proc. London Math. Soc., to appear

[Sm1] Smyth, C.J.: A Kronecker-type theorem for complex polynomials in several variables. Can. Math. Bull. 24, 447-452 (1981)

[Sm2] Smyth, C.J.: On measures of polynomiais in several variables. Bull. Aust. Math. Soc. 23, 49-63 (1981)

[T1] Thomas, R.K.: The addition theorem for the entropy of transformations of $G$-spaces. Trans. Am. Math. Soc. 160, 119-130 (1971)

[T2] Thomas, R.K.: Metric properties of transformations of $G$-spaces. Trans. Am. Math. Soc. 160, 103-117 (1971)

[W] Walters, P.: An Introduction to Ergodic Theory. Berlin-Heidelberg-New York: Springer 1982

[Yn] Young, R.M.: On Jensen's formula and $\int_{0}^{2 \pi} \log \left|1-e^{i \theta}\right| \mathrm{d} \theta$. Am. Math. Mon. 93, 44-45 (1986)

[Yz1] Yuzvinskii, S.A.: Metric properties of endomorphisms of compact groups. Izv. Akad. Nauk SSSR, Ser. Math. 29, 1295-1328 (1965); Engl. transl. Am. Math. Soc. Transl. (2) 66, 63-98 (1968)

[Yz2] Yuzvinskii, S.A.: Computing the entropy of a group endomorphism. Sib. Mat. $\hat{Z} . \mathbf{8}$, 230-239 (1967) (Russian). Engl. transl. Sib. Math. J. 8, 172-178 (1968) 